\title{
Multiple Aspects of Neural Activity during Reaching Preparation in the Medial Posterior Parietal Area V6A
}

\author{
Rossella Breveglieri, Claudio Galletti, Giulia Dal Bò, \\ Kostas Hadjidimitrakis, and Patrizia Fattori
}

\begin{abstract}
The posterior parietal cortex is involved in the visuomotor transformations occurring during arm-reaching movements. The medial posterior parietal area V6A has been shown to be implicated in reaching execution, but its role in reaching preparation has not been sufficiently investigated. Here, we addressed this issue exploring the neural correlates of reaching preparation in V6A. Neural activity of single cells during the instructed delay period of a foveated Reaching task was compared with the activity in the same delay period during a Detection task. In this latter task, animals fixated the target but, instead of performing an arm reaching movement, they responded with a button release to the go signal. Targets were allocated in different positions in 3-D space. We found three types of neurons: cells where delay activity was equally spatially
\end{abstract}

\section{INTRODUCTION}

It is well known that the cells of the posterior parietal cortex (PPC) process different types of signals, like gaze, attention, memory, and corollary motor discharges (Galletti et al., 2010; Gottlieb \& Snyder, 2010; Andersen \& Cui, 2009; Galletti, Kutz, Gamberini, Breveglieri, \& Fattori, 2003; Eskandar \& Assad, 1999; Barash, Bracewell, Fogassi, Gnadt, \& Andersen, 1991). A large amount of evidence also demonstrated that the PPC is involved in movement planning (Buneo \& Andersen, 2006; Andersen \& Buneo, 2002; Battaglia-Mayer et al., 2001; Snyder, Batista, \& Andersen, 2000; Kalaska \& Crammond, 1995). The parietal reach region (PRR), in particular, was functionally defined as an area where the intention to move the arm modulates specifically (or more strongly than other signals) the cell discharge (Snyder et al., 2000; Snyder, Batista, \& Andersen, 1997).

A visuomotor PPC area lying close to PRR, called V6A (Galletti et al., 2003; Galletti, Fattori, Kutz, \& Gamberini, 1999; see Figure 1), has been widely studied with instructed-delay reaching tasks (Hadjidimitrakis et al., 2013; Bosco, Breveglieri, Chinellato, Galletti, \& Fattori, 2010; Fattori et al., 2009; Marzocchi, Breveglieri, Galletti, \& Fattori,

University of Bologna tuned in the two tasks (Gaze cells), cells spatially tuned only during reaching preparation (Set cells), and cells influenced by both gaze and reaching preparation signals (Gaze/Set cells). In cells influenced by reaching preparation, the delay activity in the Reaching task could be higher or lower compared with the Detection task. All the Set cells and a minority of Gaze/Set cells were more active during reaching preparation. Most cells modulated by movement preparation were also modulated with a congruent spatial tuning during movement execution. Present results highlight the convergence of visuospatial information, reach planning and reach execution signals on V6A, and indicate that visuospatial processing and movement execution have a larger influence on V6A activity than the encoding of reach plans.

2008; Fattori, Kutz, Breveglieri, Marzocchi, \& Galletti, 2005; Fattori, Gamberini, Kutz, \& Galletti, 2001). These studies have shown that V6A cells are activated during both preparation and execution of reaching. It is reasonable to suppose that during arm movement preparation signals related to the planning of the impending movement do modulate V6A activity. However, because many V6A cells are modulated by direction and depth of gaze (Breveglieri, Hadjidimitrakis, Bosco, et al., 2012; Hadjidimitrakis et al., 2011; Galletti, Battaglini, \& Fattori, 1995), it is also plausible that cell modulation during fixation before reaching is because of the gaze signals instead of, or besides, the planning of impending movement. In addition, the activity during movement preparation could reflect the anticipatory activity related to the expectation of a cue (Confais, Kilavik, Ponce-Alvarez, \& Riehle, 2012; Kalaska \& Crammond, 1995) and/or the visuospatial/attentional processing related to an upcoming motor action (Baldauf \& Deubel, 2008; Baldauf, Wolf, \& Deubel, 2006; Rushworth, Nixon, \& Passingham, 1997). Although we are aware of the difficulty to separate the contribution of these different signals in cell firing, we tried to evaluate the relative contribution of gaze/ visuospatial and reach planning-related signals.

We tested single V6A cells in two task conditions: (i) a Reaching task, where the animal waited for a go signal to execute an arm-reaching movement toward foveated 


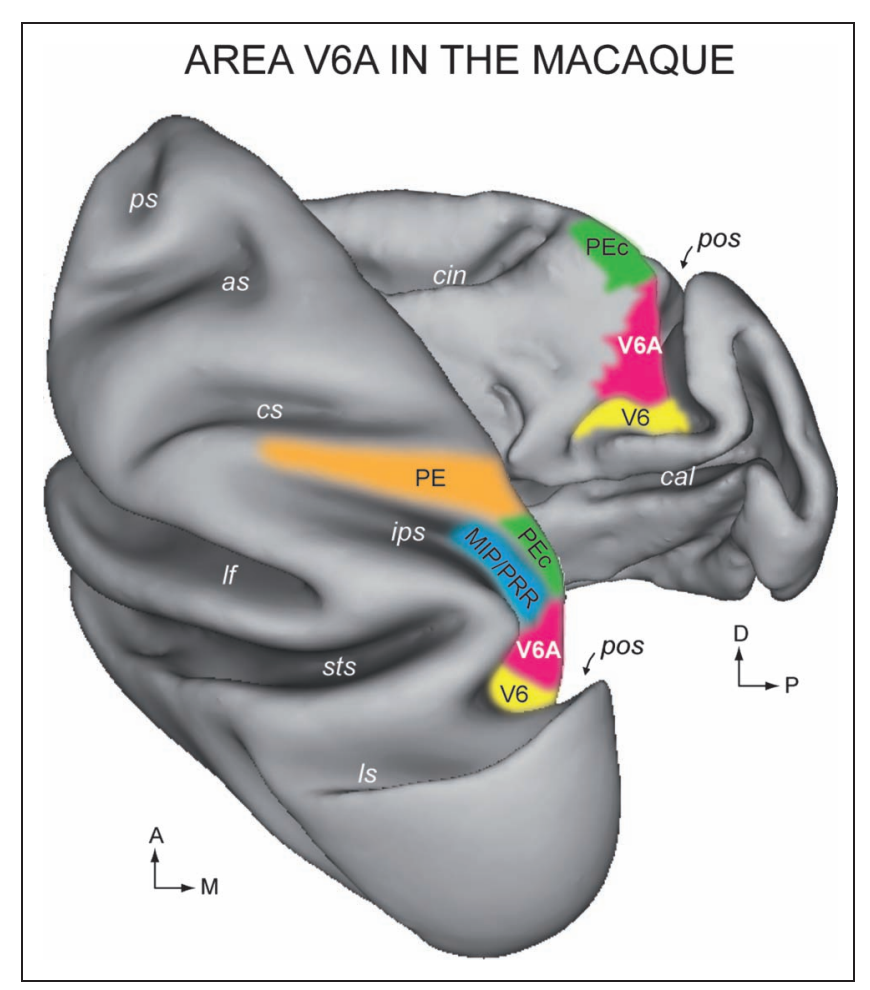

Figure 1. Anatomical location of area V6A. Dorsal (left) and medial (right) views of the macaque brain showing the locations of V6A and of nearby PPC areas: V6, area V6 (Galletti, Fattori, Gamberini, et al., 1999); V6A, area V6A (Galletti, Fattori, Kutz, et al., 1999; Galletti et al., 1996); PE, area PE (Pandya \& Seltzer, 1982); PEc, caudal area PE (Pandya \& Seltzer, 1982); MIP, medial intraparietal area (Colby \& Duhamel, 1991); PRR, parietal reach region (Snyder, Batista, \& Andersen, 1998); ps, principal sulcus; as, arcuate sulcus; cs, central sulcus; cin, cingulate sulcus; lf, lateral fissure; ips, intraparietal sulcus; sts, superior temporal sulcus; ls, lunate sulcus, cal, calcarine sulcus; pos, parieto-occipital sulcus. $\mathrm{A}=$ anterior; $\mathrm{M}=$ medial; $\mathrm{D}=$ dorsal; $\mathrm{P}=$ posterior.

targets, and (ii) a Detection task, where the animal fixated the same targets and after the go signal released a button without performing any reaching arm movement. In both task conditions, there was a delay period where the monkeys had to withhold their response until a go signal appeared that instructed them to act, but in the first task the action was a spatially targeted arm movement, whereas in the second task it was an automatic, stereotyped, and nonspatially directed arm response (button release). The comparison of delay activity in the two conditions allowed us to probe for the presence of signals related to reaching preparation.

The use of instructed-delay reaching tasks, although artificial, is a standard method for dissociating in distinct time epochs the activity related to sensory input from those related to motor output (Snyder et al., 2000). Our experimental configuration was similar to the ones used in studies of reach planning activity in area PE and in the dorsal premotor cortex (PMd) that provided stronger evidence for reaching preparation in PMd than in PE (Kalaska \& Crammond, 1995; Boussaoud \& Wise, 1993).
We found different types of cells in V6A: cells equally spatially tuned by target location, regardless of whether the target was then reached out or not; cells whose delay activity was spatially tuned only when reaching movement was then performed; and-the majority of cases-cells whose spatial tuning of delay activity was strengthened, attenuated, or abolished in the Reaching task. Most of the cells spatially tuned during reaching preparation were also spatially tuned during movement execution and showed a congruent spatial tuning in the two time epochs. Some cells were inhibited during reaching preparation; others were excited. Preliminary data were presented in abstract form (Breveglieri, Hadjidimitrakis, Dal Bò, et al., 2012).

\section{METHODS \\ Experimental Procedures}

Two male macaque monkeys (Macaca fascicularis) weighing $4.4 \mathrm{~kg}$ (Monkey A) and $3.8 \mathrm{~kg}$ (Monkey B) were used. Initially, the animals were habituated to sit in a primate chair and to interact with the experimenters. Then, a head-restraint system and a recording chamber were surgically implanted under general anesthesia (sodium thiopenthal, $8 \mathrm{mg} / \mathrm{kg} / \mathrm{h}$ iv) following the procedures reported by Galletti et al. (1995). A full program of postoperative analgesia (ketorolac trometazyn, $1 \mathrm{mg} / \mathrm{kg} \mathrm{im}$, immediately after surgery, and $1.6 \mathrm{mg} / \mathrm{kg} \mathrm{im}$, on the following days) and antibiotic care (Ritardomicina, benzatinic benzylpenicillin + dihydrostreptomycin + streptomycin, 1-1.4 ml/10 kg every 5-6 days) followed surgery. Experiments were performed in accordance with national laws on care and use of laboratory animals and with the European Communities Council Directive of November 24, 1986 (86/609/EEC) and that of September 22, 2010 (2010/63/EU). All the experimental protocols were approved by the Bioethical Committee of the University of Bologna. During training and recording sessions, particular care was taken to avoid any behavioral and clinical sign of pain or distress. Extracellular recording techniques and procedures to reconstruct microelectrode penetrations were similar to those described in other reports (e.g., Galletti, Fattori, Battaglini, Shipp, \& Zeki, 1996; Galletti et al., 1995). Single cell activity was extracellularly recorded from the anterior bank of the parieto-occipital sulcus (POs). The electrodes entered directly into the cortex of the exposed surface of the caudal aspect of superior parietal lobule or passed through the occipital pole and the POs to reach the anterior bank of the sulcus in the depth (inclination angle of electrodes was $28-30^{\circ}$ posteriorly from the coronal plane). After passing through areas V1-V2 of the occipital lobe, the electrode reached the anterior bank of the POs at a variable depth (up to $8 \mathrm{~mm}$ ) according to the anteroposterior coordinate of penetration. Area V6A was initially recognized on functional grounds following the criteria described in Galletti, Fattori, Kutz, et al. (1999) and later confirmed based on the cytoarchitectonic 
criteria of Luppino, Ben Hamed, Gamberini, Matelli, and Galletti (2005). We performed multiple electrode penetrations using a five-channel multielectrode recording system (Thomas Recording GmbH, Giessen, Germany). The electrode signals were amplified (at a gain of 10,000) and filtered (bandpass between 0.5 and $5 \mathrm{kHz}$ ). Action potentials in each channel were isolated with a waveform discriminator (Multi Spike Detector; Alpha Omega Engineering Nazareth, Israel) and were sampled at $100 \mathrm{kHz}$. Quality of single-unit isolation was determined by the homogeneity of spike waveforms and clear refractory periods in ISI histograms during spike-sorting. Only well-isolated units not changing across tasks were considered.

The animal behavior was controlled by custom-made software implemented in Labview (National Instruments, Austin, TX) environment (Kutz, Marzocchi, Fattori, Cavalcanti, \& Galletti, 2005). Eye position signals were sampled with two cameras (one for each eye) of an infrared oculometer system (ISCAN, Woburn, MA) at $100 \mathrm{~Hz}$. The vergence angle was not recorded online, but it was reconstructed offline from the horizontal eye positions of the two eyes. A sort of control for vergence resulted from the presence of electronic windows (one for each eye, $4^{\circ} \times 4^{\circ}$ each) that controlled the frontoparallel gaze position, so that we could set an offset of the horizontal eye position signal for targets located in the same direction, but at different depths.

\section{Histological Reconstruction of the Recording Sites}

At the end of electrophysiological recordings, a series of electrolytic lesions ( $30 \mu \mathrm{A}$ cathodic pulses for $30 \mathrm{sec}$ ) were performed at the limits of recorded region. Then, animals were anesthetized with ketamine hydrochloride $(15 \mathrm{mg} / \mathrm{kg} \mathrm{im})$ followed by an intravenous lethal injection of sodium thiopental. The animals were perfused through the left cardiac ventricle with the following solutions: $0.9 \%$ sodium chloride, $3.5-4 \%$ paraformaldehyde in $0.1 \mathrm{M}$ phosphate buffer, $\mathrm{pH} 7.4$, and $5 \%$ glycerol in the same buffer. Brains were then removed from the skull, photographed, placed in 10\% buffered glycerol for 3 days and in 20\% glycerol for 4 days, and cut on a freezing microtome at $60 \mu \mathrm{m}$ in parasagittal plane. One section every five was stained with the Nissl method (thionin, $0.1 \%$ in $0.1 \mathrm{M}$ acetate buffer, $\mathrm{pH} 3.7$ ) for cytoarchitectonic analysis. Procedures to reconstruct microelectrode penetrations and to assign neurons recorded in the anterior bank of the POs to area V6A were the same as previously described by our group (Galletti, Fattori, Gamberini, \& Kutz, 1999; Galletti, Fattori, Kutz, et al., 1999; Galletti et al., 1996). Briefly, electrode tracks and approximate location of each recording site were reconstructed on histological sections of the brain on the basis of several cues, such as electrolytic lesions, coordinates of penetrations within recording chamber, cortical areas passed through before reaching the ROI, and depths of passage points between gray and white matter. All neurons were assigned to the dorsal or ventral sectors of area V6A following the criteria defined by Luppino et al. (2005) and described in detail in a recent work (Gamberini, Galletti, Bosco, Breveglieri, \& Fattori, 2011).

\section{Behavioral Tasks}

Electrophysiological signals were collected while the monkeys were performing two tasks: an instructed-delay body-out reaching task (the "Reaching task") and a Detection task. In both tasks, the targets were allocated in different positions in the 3-D space. The two tasks were identical except for the fact that in Reaching task the animal was fixating a target that it would reach when instructed, whereas in Detection task the monkeys fixated the target waiting for the go signal to release a button, not being required nor allowed to perform reaching movements.

In both tasks monkeys sat in a primate chair, with the head restrained, and faced a horizontal panel located at eye level. Nine light-emitting diodes (LEDs) mounted on the panel at different distances from the eyes were used as fixation and reaching targets (Figure 2A, left). As shown in the right part of Figure 2A, the target LEDs were arranged in three rows: one central, along the sagittal midline, and two lateral, at isoversion angles of $-15^{\circ}$ and $+15^{\circ}$, respectively. Along each row, three LEDs were located at isovergent positions of $17.1^{\circ}, 11.4^{\circ}$, and $6.9^{\circ}$, respectively. The two animals had the same interocular distance $(3.0 \mathrm{~cm})$, so we placed the isovergent rows at the same distance from the monkeys in both animals (nearest targets: $10 \mathrm{~cm}$; intermediate targets: $15 \mathrm{~cm}$; far targets: $25 \mathrm{~cm}$ ). The range of vergence angles was chosen to be within the limits of peripersonal space, so the monkeys were able to reach all target positions.

The animals performed the tasks with the limb contralateral to the recording site while mantaining steady fixation. The hand started the trial pushing a button (home button, $2.5 \mathrm{~cm}$ in diameter, HB in Figure 2A) placed outside the monkeys' visual field, $5 \mathrm{~cm}$ in front of its trunk.

In the Reaching task (Figure 2B), 1000 msec after home button pressing one of the nine LEDs lit up green. The monkeys were required to fixate the fixation point while keeping the button pressed. The fixation point served as a cue concerning the direction of the arm movement to perform. However, the monkeys needed to withhold the instructed behavior without performing any eye or arm reaching movement for $1700-2500 \mathrm{msec}$, till the change in color of fixation LED (green to red). The color change of fixation target was the go signal for the animal to release the home button and start an arm movement toward the target. The monkeys had 1 sec after the go signal to reach the target; otherwise, the trial was aborted. Then, monkeys pushed the target and held the hand on it for 800-1200 msec. The target offset cued the monkeys to release the LED and return to the home button, which ended the trial and allowed monkeys to receive reward. 


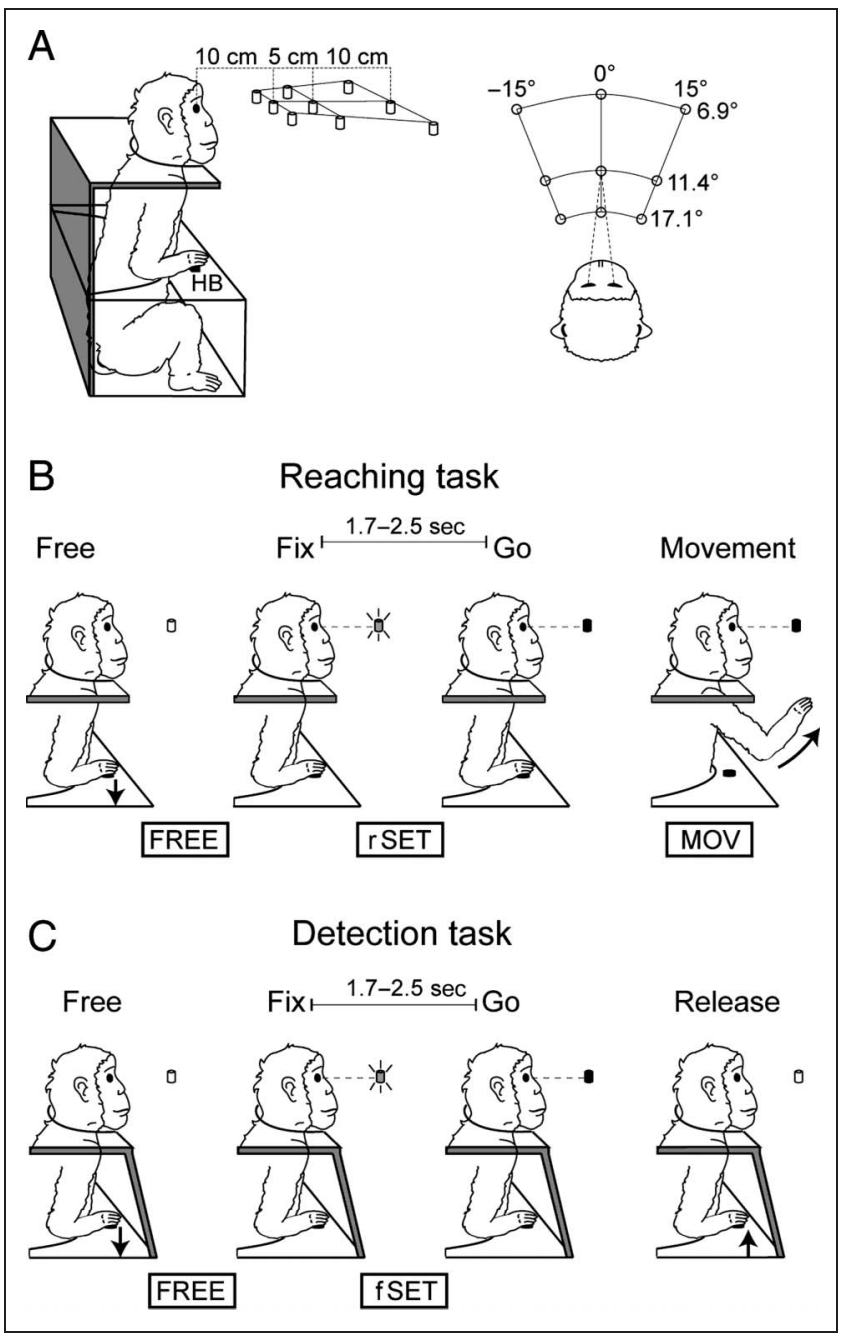

Figure 2. Experimental setup and schematic representation of the tasks. (A) Scheme of the setup used for the Detection and Reaching tasks. Exact distances are indicated in the lateral (left) and top (right) views. Nine LEDs are used as fixation and reaching targets, embedded in a panel located at eye level. $\mathrm{HB}=$ home button. (B, C) Time courses and behavioral epochs in the Reaching (B) and Detection tasks (C). Black arrows indicate actions performed in the two tasks.

During the recording sessions, the animals had an average performance of about $85 \%$ correct trials. Fixation had to remain stable on target throughout the trial; otherwise, trial was aborted. Arm-reaching movements were always directed toward a foveated target. Therefore, the retinotopic coordinates of reaching targets remained constant throughout the task, whereas the direction and distance of movement relative to the initial hand position (home button) changed trial by trial according to target position.

In the Detection task (Figure 2C), a Plexiglass wall was mounted on the chair to prevent arm reaching movements. As for the Reaching task, a trial began when monkeys pressed the home button. After $1000 \mathrm{msec}$, one of the nine LEDs lit up green. The animal was required to fixate the LED and wait for 1700-2500 msec for a change in color of fixation LED (green to red) without performing any eye or arm movement; otherwise, trial was automatically aborted. When the LED color changed from green to red (go signal), the monkeys had to release the button to receive the reward. No reaching movements were required in this task.

The Detection and the Reaching tasks were run separately for each cell. The Plexiglass wall clearly informed the monkeys about the task to be performed. At the initial stages of the training in the Detection task, the monkeys sometimes bumped into the Plexiglass wall. After being well trained in alternating between the two tasks, they quickly associated the placement/removal of the wall with the task that they had to perform. As a result, during recording sessions, they never confused reach with detection trials. Each task was a sequence of 90 trials, 10 for each LED position. Positions were randomized trial by trial within the block of 90 trials. Both tasks were performed in darkness. The background light was switched on for a few minutes before starting a new task to reduce dark adaptation. To further minimize the role of vision during reaching, the brightness of the LED was strongly reduced, so it was barely visible during the task. The overall performance was monitored by a Labview software supervisor system (see Kutz et al., 2005), which checked the status of microswitches (monopolar microswitches, RS Components, Northans, UK) mounted under the home button and LED buttons. Presses/releases were checked with 1-msec resolution. Monkeys' arm movements were continuously video-monitored by means of miniature, infrared illumination-sensitive videocameras. The movement strategy (i.e., orientation and position of arm/hand, finger postures) during Reaching and Detection tasks was estimated using video images at 25 frames/sec. We analyzed offline the single video frames to compare animals' behavior during the tasks.

\section{Data Analysis}

Data analysis was performed using Matlab (The Mathworks, Natick, MA).

As the two tasks were run in separate blocks, we carefully checked online spike waveform and constancy of background neural activity in the two conditions. Background activity (epoch FREE) was the neural activity occurring in the last $300 \mathrm{msec}$ before LED onset. If spike waveform or background activity (or both) changed across conditions, we stopped data collection and discarded the acquired data.

The delay activity was quantified as the firing rate during the last 500-msec before the go signal (epoch called rSET in the Reaching task and fSET in the Detection task; see Figure 2B, C). The duration and time of occurrence of these epochs were chosen in agreement with the literature in this field (e.g., Batista et al., 2007). In both SET epochs, we excluded the initial part of the fixation time to avoid contamination from visual and saccadic signals as well as from the transient part of gaze-related discharge (Breveglieri, Hadjidimitrakis, Bosco, et al., 2012; 
Hadjidimitrakis et al., 2011; Kutz, Fattori, Gamberini, Breveglieri, \& Galletti, 2003; Nakamura, Chung, Graziano, \& Gross, 1999). Because we found that for the overwhelming majority of neurons in our population the SET activity was not statistically different in trials with different durations of the delay period (Student's $t$ test, $p>.05$ ), we grouped together the trials with different delay durations.

To assess spatial modulation of cell activity during fSET and rSET, we performed a one-way ANOVA (factor: spatial position of the target, nine levels) in which the dependent variable was the firing rate. A Student's $t$ test was used to compare the overall activity of each cell in rSET with that of fSET. Significance was set at $p<.05$. We classified the cells as follows. "Gaze" cells: cells that were spatially tuned in fSET and rSET with a congruent spatial tuning (assessed as explained in the next paragraph) and in which SET activity was not statistically different between the two tasks (Student's $t$ test, $p>.05$ ); "Set" cells: cells where tuning was present only in rSET; "Gaze/Set" cells: cells either tuned in both tasks, with SET activity that was statistically different in the two tasks (Student's $t$ test, $p<.05$ ) or cells with tuning present only in fSET. In "Gaze/Set" cells, reach preparation could have an excitatory or inhibitory effect on SET activity (rSET $>$ fSET and rSET $<$ fSET, respectively).

The cell discharge during movement execution in Reaching task was quantified as the mean frequency of discharge in an epoch (MOV; see Figure 2B) calculated from 200 msec before movement onset to the movement end, as detected by button release and target pushing, respectively. The epochs rSET and MOV never overlapped, as rSET ends at the go signal, MOV starts $200 \mathrm{msec}$ before the movement onset, and the mean time from the go signal to the movement onset is $283 \pm 32 \mathrm{msec}$. Movement times in reaching trials were calculated as the time difference between home button release and target LED press, as detected by presses/releases of the microswitches.

\section{Congruency of Spatial Tuning in Reaching Preparation and Execution}

To analyze the congruency of spatial tuning of cell activity during different epochs (rSET and MOV, rSET and fSET), a multilinear regression model was applied using the Matlab function "regress." The model equation for the firing rate was

$$
A\left(X_{i}, Y_{i}\right)=b_{0}+b_{1} X_{i}+b_{2} Y_{i}
$$

where $A$ was the neural activity in spikes per second for the $i$ th trial. $X_{i}$ and $Y_{i}$ are the positions of target defined as eye vergence and version angles, respectively, and as $b_{1}$ and $b_{2}$ are regression coefficients and $b_{0}$ is the intercept. After being tested for their significance (i.e., when their confidence intervals were not crossing zero), the coefficients were normalized with their standard deviation. These coefficients were then used to determine the spatial preference in cells with a significant main effect (ANOVA $p<.05$ ) in both epochs rSET and MOV and for Gaze cells (rSET and fSET). Moreover, we compared the coefficients obtained with the activities of different epochs (or of different tasks) to study the consistency of spatial tunings across epochs/tasks. We considered the spatial tuning in different epochs congruent if the correlation coefficients during the epochs had the same sign.

To quantify the selectivity of neurons for different spatial positions, we computed a preference index (PI) based on the magnitude of neuronal response to each of the nine target positions. According to Moody and Zipser (1998), it was computed as follows:

$$
\mathrm{PI}=\frac{n-\left(\frac{\sum a_{i}}{a_{\mathrm{pref}}}\right)}{n-1}
$$

where $n$ is the number of target positions, $a_{i}$ is the activity for position $i$, and $a_{\text {pref }}$ is the activity for the preferred position. The PI can range between 0 and 1 . A value of 0 indicates the same magnitude of response for all positions, whereas a value of 1 indicates a preference for only one position. We calculated the PI in the cells spatially tuned during reach preparation and execution. To compare the PI of a cell during different conditions, confidence intervals on PIs were estimated using a bootstrap test. Synthetic response profiles were created by drawing $N$ firing rates (with replacement) from the $N$ repetitions of experimentally determined firing rates. The PI was recomputed using these $N$ firing rates. Ten thousand iterations were performed, and confidence intervals were estimated as the range that delimited 95\% of the computed indices (Batista et al., 2007). In the final plots (see Figure 8A), filled points represent cells whose confidence intervals cross the unity diagonal. This means that the amount of selectivity (expressed as PI) during the two conditions was not statistically distinguishable.

For each cell, we estimated the preference for specific target spatial positions by calculating a mean vector of discharge rate for each neuron during rSET and MOV (Mardia, 1972). We weighted the polar coordinates of each tested position ( $R_{i}$, distance; $\alpha_{i}$, angle) with the mean activity recorded in that position $\left(A_{i}\right)$ normalized by the sum of all activities in that epoch $\left(A_{\mathrm{tot}}=\Sigma_{i} A_{i}\right)$ as follows:

$$
\begin{aligned}
& R_{\text {pref }}=\sum_{i} R_{i} \times\left(\frac{A_{i}}{A_{\mathrm{tot}}}\right) \\
& \alpha_{\text {pref }}=\sum_{i} \alpha_{i} \times\left(\frac{A_{i}}{A_{\mathrm{tot}}}\right)
\end{aligned}
$$

For this purpose, the coordinates of each tested position were expressed in a polar coordinate system whose origin was midway between the animal's eyes. 
We measured the shift of the preferred position occurring between preparation and execution of arm movement by subtracting the coordinates of preferred position between the two time epochs as follows:

$$
\begin{aligned}
& \Delta R=R_{\text {pref }} \mathrm{MOV}-R_{\text {pref }} \mathrm{SET} \\
& \Delta \alpha=\alpha_{\text {pref }} \mathrm{MOV}-\alpha_{\text {pref }} \mathrm{SET}
\end{aligned}
$$

where $R_{\text {pref }} \mathrm{MOV}, \alpha_{\text {pref }} \mathrm{MOV}$ are the polar coordinates of preferred position during reach execution (MOV) and $R_{\text {pref }} \mathrm{SET}, \alpha_{\text {pref }} \mathrm{SET}$ are the polar coordinates of preferred position during reach preparation (rSET). The shift could range from $\left(0 ; 0^{\circ}\right)$, in case the preferred position was the same in the two conditions, to $\left(14.91 ; 30^{\circ}\right)$, in case the preferred positions were located in opposite parts of the space (i.e., in the far-contralateral position vs. the near-ipsilateral one or vice versa). This approach allowed us to identify neurons that did not change the preferred position between movement preparation and execution. Such cells would have both $\Delta R$ and $\Delta \alpha$ very low and statistically indistinguishable from zero. To find the significance level within which a cell did not exhibit significant spatial difference between the two epochs, trials were randomly resampled without replacement and the computation procedure of $\Delta R$ and $\Delta \alpha$ was repeated (10,000 iterations). We determined a border that encompassed $95 \%$ of the values (dashed line in Figure $8 \mathrm{~B}$ ). This border was defined by the line $R+\alpha=0.39$. Neurons with similar preferred positions during reaching preparation and execution lie inside this border (i.e., $R+\alpha<$ $0.39, p>.05$ ), meaning that their preferred positions in these task stages were not statistically distinguishable from zero.

We characterized the neurons modulated by movement preparation and execution using a Preparation-Execution Index (PEI) calculated from the movement preparation and execution responses during the Reaching task. The movement preparation response was defined as the mean activity during rSET; the movement execution response was defined as the mean activity during MOV. The PEI = (execution - preparation)/(execution + preparation) was calculated for each neuron, for each target position and then averaged across positions to have one value for each cell. The PEI can range from -1 to 1 , with positive/negative values indicating that the neuronal response during movement execution increased/decreased with respect to movement preparation.

For each cell, a spike density function (SDF; Gaussian kernel, half-width $40 \mathrm{msec}$ ) was calculated for each trial and then averaged across all the trials referred to a given LED and then across all the conditions. In each neuron, the maximum discharge frequency in the SET epoch or in MOV epoch was used to normalize the SDFs. Population SDFs were constructed by averaging the individual SDFs of all the cells (Marzocchi et al., 2008).

\section{Timing of Task-related Delay Activity Modulations}

To define in each cell the time when delay activity started to differ between the two tasks, we applied a sliding window procedure (window size $=100 \mathrm{msec}$; step size $=$ $10 \mathrm{msec})$ that allowed us to recognize the onset of activity modulation in the delay period. For each neuron, we looked for the first of (at least) 10 consecutive 100-msec bins, calculated backward from the go signal, in which the response of the cell during Reaching trials was significantly different from that during Detection trials (Student's $t$ test, $p<.05)$. This analysis was carried out only in trials with equal duration and by pooling together the activity of all tested positions in that cell.

This analysis gave precise information about the time course of modulations but was pretty sensitive to noise and to the transient fluctuations of neural discharge across trials that are inherent in biological responses and became more prevalent when small time intervals were considered. This variability, in conjunction with our selection criteria (that is at least 10 consecutive time bins including the last one before the go signal had to be significantly different in the two tasks), was the reason why many cells were excluded from this type of analysis. To resolve this issue, we also adopted an approach less sensitive to noise by computing the onset of the difference in neural activity quantified as SDF. To this purpose, an SDF was calculated for each task of each cell, using a Gaussian kernel with half width (standard) of $40 \mathrm{msec}$, averaging across all the available repetitions in each task. The onset of the difference between neural activity in Detection and Reaching tasks in each cell was computed using a custom-made algorithm based on pairwise sliding windows permutation test (see Hadjidimitrakis et al., 2011; window size $=100 \mathrm{msec}$; step size $=20 \mathrm{msec}$ ) analyzing the last $1700 \mathrm{msec}$ before the go signal. A similar approach was also used by Battaglia-Mayer, Mascaro, and Caminiti (2007). This approach enabled us to recruit the overwhelming majority of cells.

\section{RESULTS}

We recorded the activity of 162 V6A neurons from two macaque monkeys (100 cells from Monkey A and 62 cells from Monkey B) trained to perform both Detection and Reaching tasks in darkness. In both tasks, during the delay before go signal, monkeys fixated a position in space under the same visual conditions (in darkness). In Reaching task, but not in Detection task, the animals also planned an arm reaching movement. According to our videobased estimation, the two animals employed the same strategy (orientation and position of arm/hand in space, finger postures) while reaching and pressing the target.

We found three types of cells (see Table 1): (i) Gaze cells that showed congruent spatial tuning of SET activity in the two tasks with no statistical difference of SET activity between the tasks; in other words, these cells were 
Table 1. Functional Classification of V6A Neurons

\begin{tabular}{lccc}
\hline Cell Type & $\begin{array}{c}\text { Animal A } \\
\text { Number (\%) }\end{array}$ & $\begin{array}{c}\text { Animal B } \\
\text { Number (\%) }\end{array}$ & $\begin{array}{c}\text { Total } \\
\text { Number (\%) }\end{array}$ \\
\hline Gaze & $29(29)$ & $12(19)$ & $41(25)$ \\
Set & $15(15)$ & $12(19)$ & $27(17)$ \\
Gaze/Set & $44(44)$ & $27(44)$ & $71(44)$ \\
None & $12(12)$ & $11(18)$ & $23(14)$ \\
Total & $100(100)$ & $62(100)$ & $162(100)$ \\
\hline
\end{tabular}

spatially tuned by the direction and/or depth of gaze during target fixation regardless of whether the target would have then been reached out or not. (ii) Set cells, which were spatially tuned in the delay only before Reaching and not in the Detection task. (iii) Gaze/Set cells, which showed different average discharge between fSET and rSET and were influenced by both gaze position and movement preparation. As shown in Table 1, results from the two monkeys were not statistically different (chi-square test, $p<.05$ ), and therefore, we will present them jointly.

\section{Gaze Cells}

A total of 41 of 162 cells (25\%) were spatially tuned during both Detection and Reaching tasks (one-way ANOVA, $p<.05)$ and showed SET activity not statistically different in the two conditions (Student's $t$ test, $p>.05$ ), so that the modulation is to be fully ascribed to the change in gaze position. As the gaze modulation in V6A is not a novel finding and was thoroughly studied in previous articles (Breveglieri, Hadjidimitrakis, Bosco, et al., 2012; Hadjidimitrakis et al., 2011; Galletti et al., 1995), this type of cells (Gaze cells) was not further analyzed in the present work.

\section{Set Cells}

The Set cells represented the $17 \%$ of our V6A population. They were spatially tuned during SET epoch in Reaching task, but not in Detection task. We therefore suggest that this activity was an expression of reaching preparation.

Figure 3 shows one example of a Set cell. It was strongly tuned (ANOVA, $p<.05$ ) during preparation (SET) and execution (MOV) of reaching in Reaching task (blue histograms), especially for reaching far-contralateral targets. One could argue that the discharge in the rSET epoch is because of the fixation of target (gaze signal), but the same cell discharged weakly and was not spatially tuned during fSET epoch (ANOVA, $p>.05$ ) in Detection task (red plots), where fixation of the target was identical (see eye traces). This cell was spatially tuned by reaching preparation and execution, but not by gaze fixation per se. Moreover, the
Figure 3. Set neuron. Neural responses to fixation and reaching of the nine LEDs. Responses are shown as peristimulus time histograms (PSTHs). Eye traces (upper trace is version, Vers; lower trace is vergence, Verg) and PSTHs are aligned at the go signal. Responses during Detection task (red PSTH) and Reaching task (blue PSTH) are superimposed. The neural responses to the nine LEDs are arranged from near (bottom) to far (top) and from contralateral (left) to ipsilateral (right) target rows with respect to the recording hemisphere. Vertical scale bars, 145 spikes/sec; calibration for version (Vers) and vergence (Verg) traces, $100^{\circ}$ and $20^{\circ}$, respectively. The duration of the three behavioral epochs (FREE, SET, MOV) are indicated below PSTHs. This neuron is strongly spatially tuned during reaching preparation and execution, especially for far-contralateral targets while discharges weakly during Detection task.

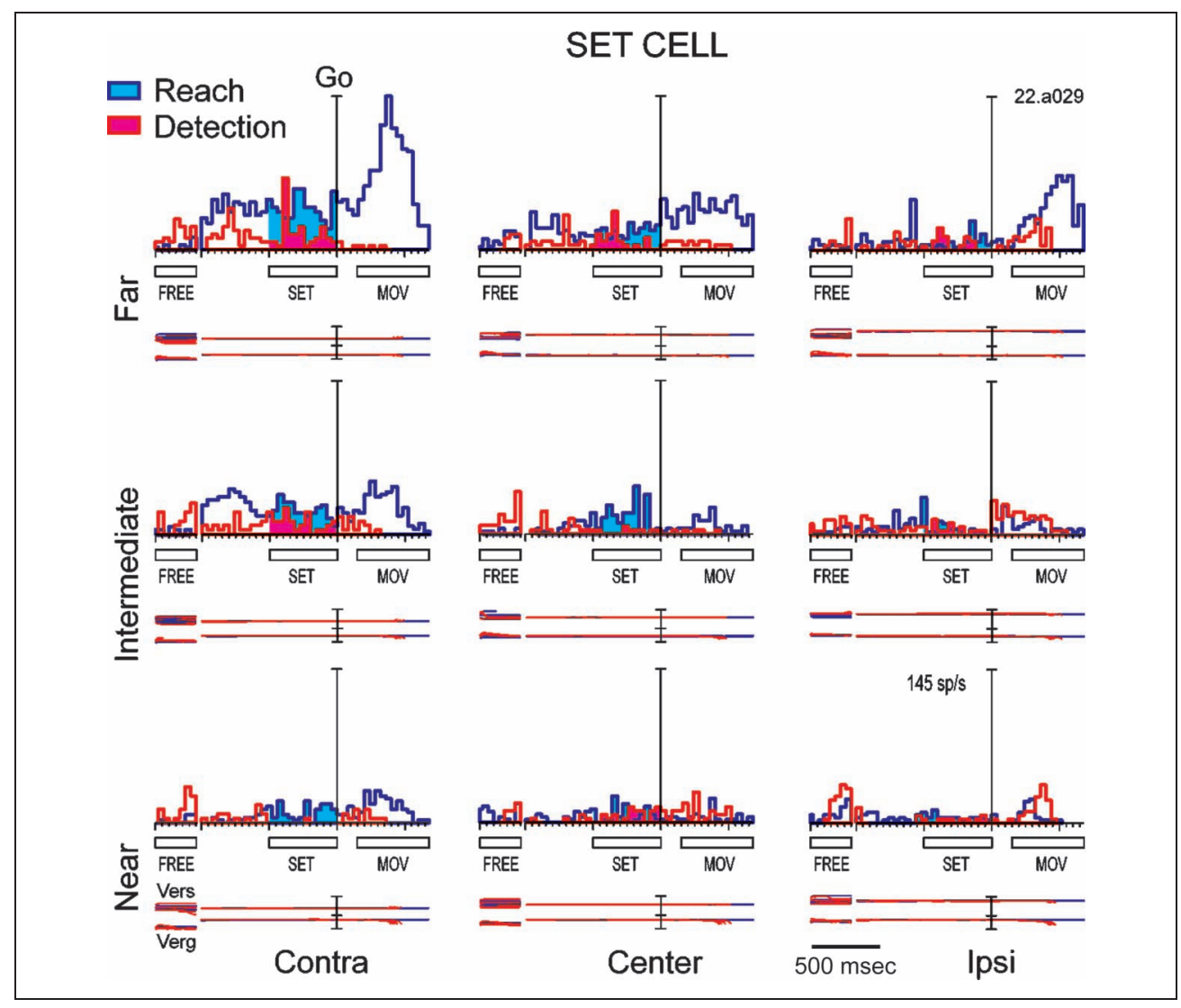




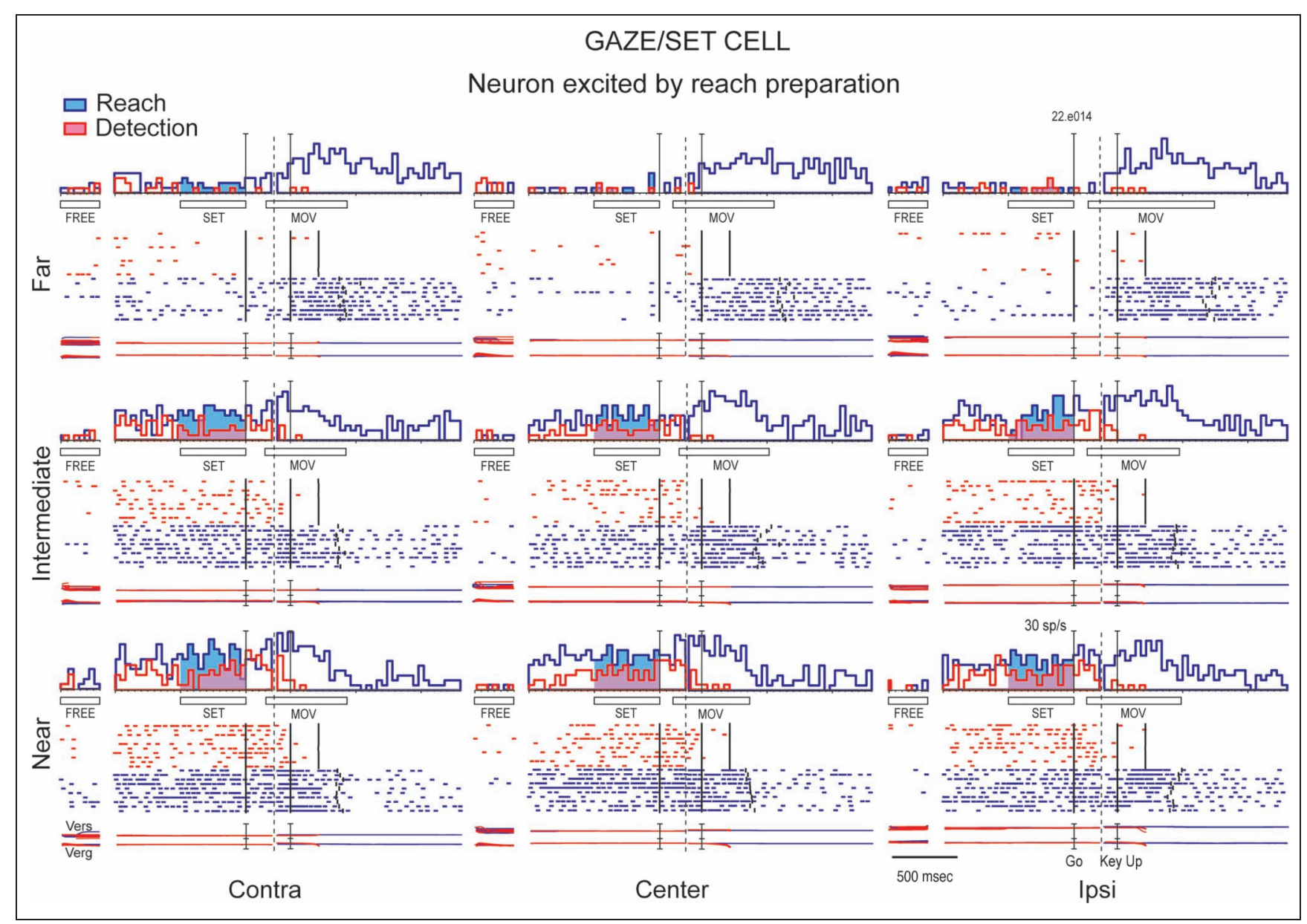

Figure 4. Excited Gaze/Set neuron. Neural activities during fixation of the nine LEDs in Reaching and Detection tasks. From top to bottom, for each panel: PSTHs of Detection (red) and Reaching (blue) trials superimposed, raster displays of impulse activity and recordings of eye movements. The short vertical ticks in rasters are spikes. Rasters are aligned twice, at the go signal and at the arm movement onset. Vertical scaling on histogram, 30 spikes/sec. Other conventions are as in Figure 3. This Gaze/Set neuron prefers near targets in both tasks. Note that the discharge during rSET is stronger than during fSET.

spatial tuning during rSET and MOV was congruent (the correlation coefficients during rSET and MOV had the same sign), supporting the view that rSET activity represents the preparation of impending reaching movement.

\section{Gaze/Set Cells}

Gaze/Set cells were the more represented type of cells in V6A (44\%; 71/162). They showed a spatial tuning in the Detection task, which was strengthened, attenuated, or completely abolished in the Reaching task. Twenty-six of 71 Gaze/Set cells (37\%) were spatially tuned during fSET, but not during rSET. These cells represent an extreme case of interactions between gaze/visuospatial and reach preparation signals. It is possible that a general arousal/alertness signal related to the preparation of the upcoming reaching action affects the neural activity of these cells, resulting in the absence of spatial tuning in the Reaching task. This as well as alternative interpretations of this particular pattern of neural modulation are discussed in more detail in the Discussion. The majority of Gaze/Set cells $(45 / 71,63 \%)$ were spatially tuned by both gaze position and reach preparation. The example of Figure 4 shows a cell more excited in the Reaching task and strongly modulated by vergence. The cell firing rate was spatially tuned during fixation in Detection task (red histograms and rasters) with the rate of discharge clearly higher when the animal gazed near targets. In Reaching task (blue histograms and rasters), the cell showed a similar spatial tuning, but the discharge during delay before reaching movement was stronger than during Detection task (Student's $t$ test, $p<.05$ ). Notice that the activity preceding LED onset (epoch FREE) was similar in the two blocks of trials, and the gaze directions and depths were identical in the delay period of the two tasks. Therefore, the higher activity during fixation in Reaching task (rSET) has to be ascribed to the preparation of arm reaching movement.

Whereas the cell in Figure 4 was influenced by vergence, the cell shown in Figure 5 was modulated by version, showing higher activity when the animal gazed ipsilateral and central locations in the visual field (see red rasters and 


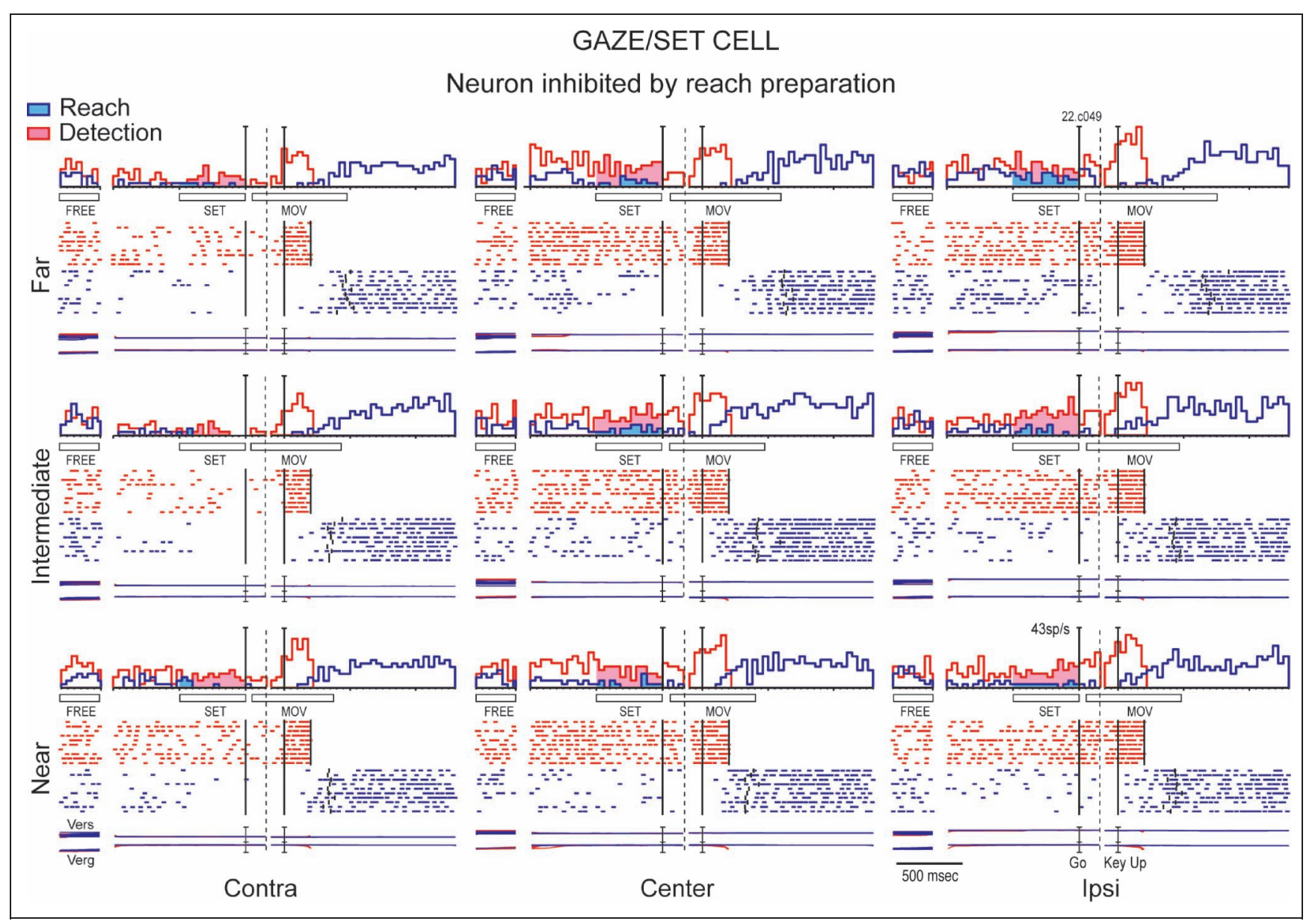

Figure 5. Inhibited Gaze/Set neuron. Neural activities during fixation of the nine LEDs in Reaching and Detection tasks. Vertical axis, 43 spikes/sec. Other conventions are as in Figures 3 and 4 . This Gaze/Set neuron is inhibited during fSET (red) and during rSET (blue), especially for the contralateral targets. The inhibition is most evident during reach preparation.

histograms). It is worth noticing that while during epoch FREE, at the beginning of the trial, the cell activity in the two tasks was not statistically different (Student's $t$ test, $p<.05)$, it resulted different in epoch SET, being the cell inhibited during the preparation of arm reaching movement (rSET, blue rasters and histograms).

Among the Gaze/Set cells, 31\% (22/71) increased the activity, as the cell of Figure 4, whereas 69\% (49/71) decreased their activity during reach preparation, as the cell of Figure 5.

\section{Population Activity}

The behavior of single cells showed in Figures $3-5$ is confirmed at population level, as it is evident in Figure 6. The population discharge of Set cells is higher in the delay period of Reaching task (blue line in Figure 6A) than in Detection task (red line in Figure 6A). The activity progressively increases during SET in Reaching task while approaching the time of the go signal, whereas it remains more or less constant, and below the level of Reaching task in the Detection trials. As to the Gaze/Set cells is concerned (Figure 6B), the population activity during SET is

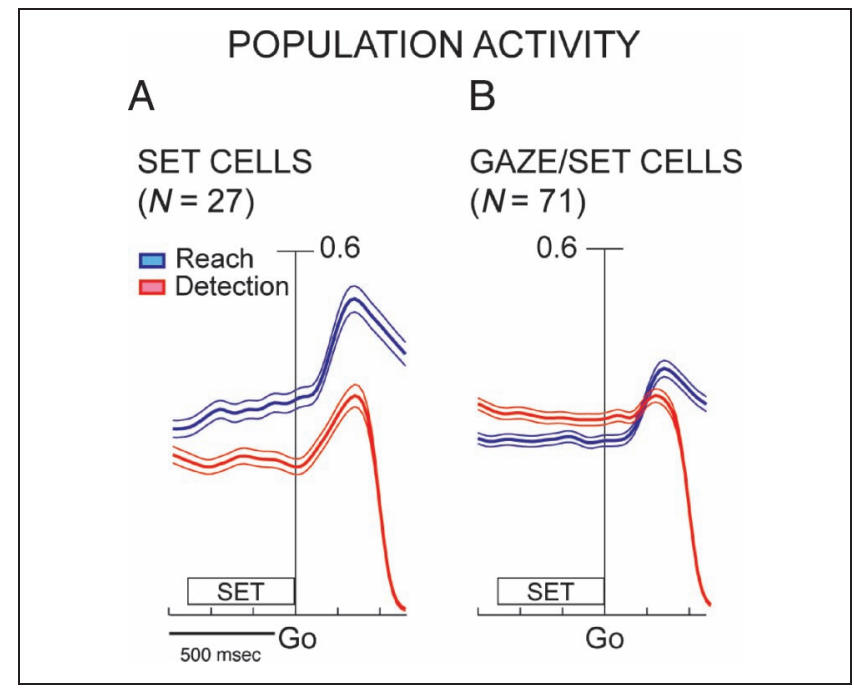

Figure 6. Population average activity in the Set and Gaze/Set cells. (A and B) Average normalized SDFs for each defined subpopulation. For each cell category, the average SDFs for the Reaching trials (blue) and for Detection trials (red) are plotted aligned on go signal (vertical line). Thick and thin solid curves represent the population average and standard errors, respectively. Scale bar in all SDF plots: 60\% of normalized activity. 
lower in Reaching than in the Detection task because of the prevalence of inhibited neurons in Reaching task, as the neuron shown in Figure 5.

We checked whether the cells belonging to the different classes were grouped within area V6A or spread all over the recorded region. As shown in Figure 7, the different types of cells (Gaze, Set, Gaze/Set) were intermingled within area V6A. In addition, Figure 7 shows that also the cells inhibited and those excited during reaching preparation were not spatially segregated within our recording site.

We estimated the effect of target direction (version) and distance (vergence) on delay activity (rSET) in the different categories of V6A cells, that is Gaze, Set, Gaze/Set cells (two-way ANOVA; factors: Distance and Direction; $p<$ $.05)$. Results are summarized in Table 2 . The majority of cells in each category were modulated by both version and vergence, in agreement with what we have reported in a very recent article (Breveglieri, Hadjidimitrakis, Bosco, et al., 2012). The individual contribution of version and vergence was similar in Gaze/Set cells, but vergence alone was more present than version alone in Set cells and in Gaze cells.

\section{Relationships between Reaching Movement Preparation and Execution}

The above-mentioned data demonstrated that about 60\% of tested cells were affected by reaching preparation (Set and Gaze/Set cells). As it is well known that most V6A cells are modulated by reaching movements (Bosco et al., 2010; Marzocchi et al., 2008; Fattori et al., 2001, 2005), also when performed in depth (Hadjidimitrakis et al., 2013), a question arises about whether this modulation is because of signals directly related to the impending arm movement, as the planning of action. If this were the case, a cell spatially tuned during both preparation and execution of reaching movement should have similar tuning in the two epochs both in strength and in spatial preference. To test this hypothesis, we first ran a oneway ANOVA (factor: Spatial Position of the target, $p<$ .05 ) on the activity in epoch MOV for cells influenced by reach preparation. Our results showed that the vast majority of these cells $(76 / 98,78 \%)$ were tuned in both reach preparation and reach execution epochs. Then, we compared the strength of the two spatial tunings (Figure 8A). Filled points in Figure 8A indicate neurons that showed a similar degree of selectivity between movement preparation and execution (the bootstrap-estimated confidence intervals cross the unity diagonal). For these neurons, we can assert with confidence that the neuron is similarly spatially sensitive during reaching preparation and execution. This was true for the majority of cells $(58 / 76,76 \%)$. The white points in Figure 8A represent cells that showed a significantly different degree of selectivity between movement preparation and execution. For example, white points below the diagonal represent cells that are more selective during reaching preparation than execution. The opposite was true for the few white points above the diagonal. As evident, white points are a minority. Overall, inhibited and excited cells were equally distributed below and above the diagonal.
Figure 7. Anatomical reconstruction of the recording sites. (A) Posterolateral view of a partially dissected macaque brain (modified from Galletti et al., 1996). The inferior parietal lobule of the right hemisphere has been cut away at the level of the fundus of the intraparietal sulcus to show the cortex of the medial bank of this sulcus. The occipital lobe of the same hemisphere has been cut away at the level of the fundus of the POs and lunate sulcus to show the cortex of the anterior bank of the POs. The medial surface of the left hemisphere is drawn to show the location on it of V6Ad (dorsal area V6A) and V6Av (ventral area V6A). (B) Flattened map of the superior parietal lobule. Each colored dot indicates the location of neurons belonging to the different categories reported in Table 1 (Set cell, Gaze cell, Gaze/Set cell, $+=$ excited, $-=$ inhibited). Other conventions as in Figure 1. Recording site involves both dorsal and ventral aspects of V6A (both V6Ad and V6Av; Gamberini et al., 2011) without any functional segregation.

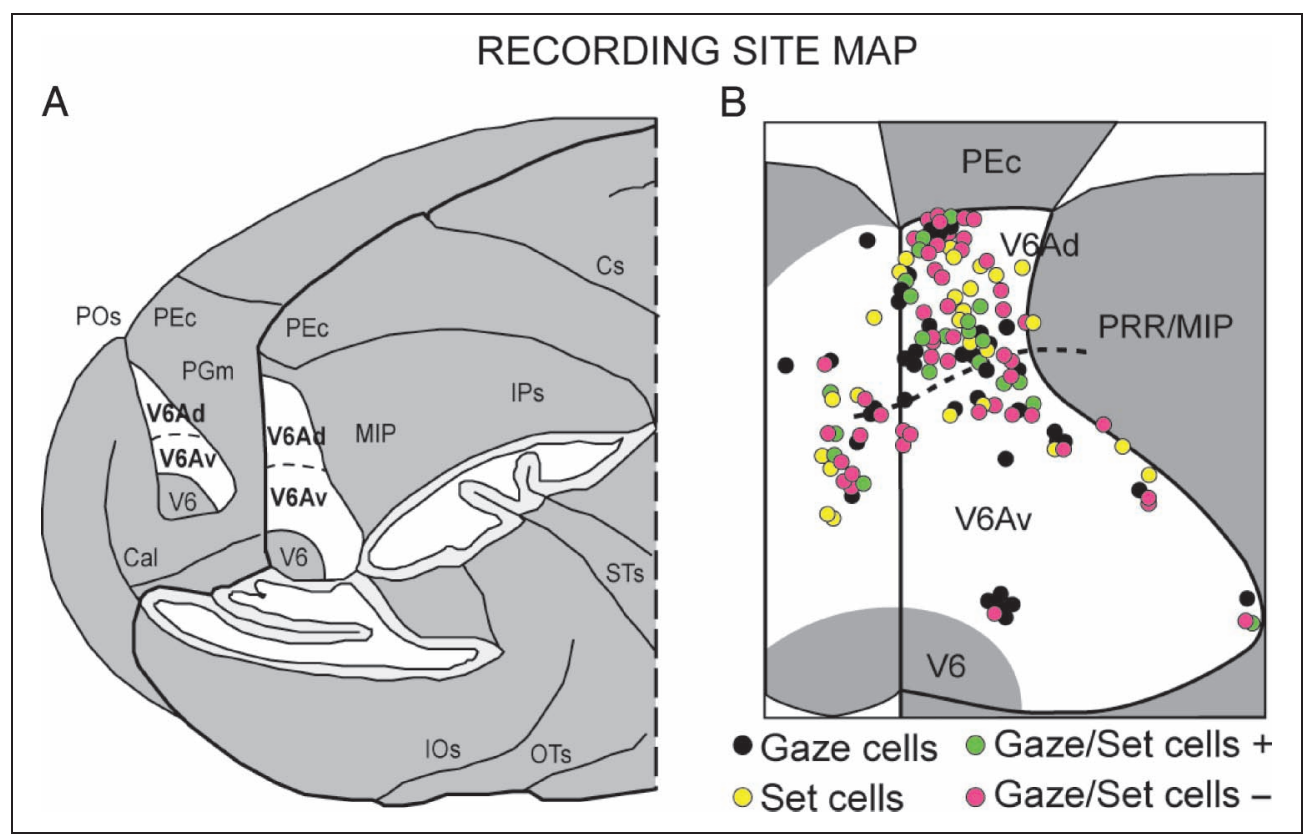




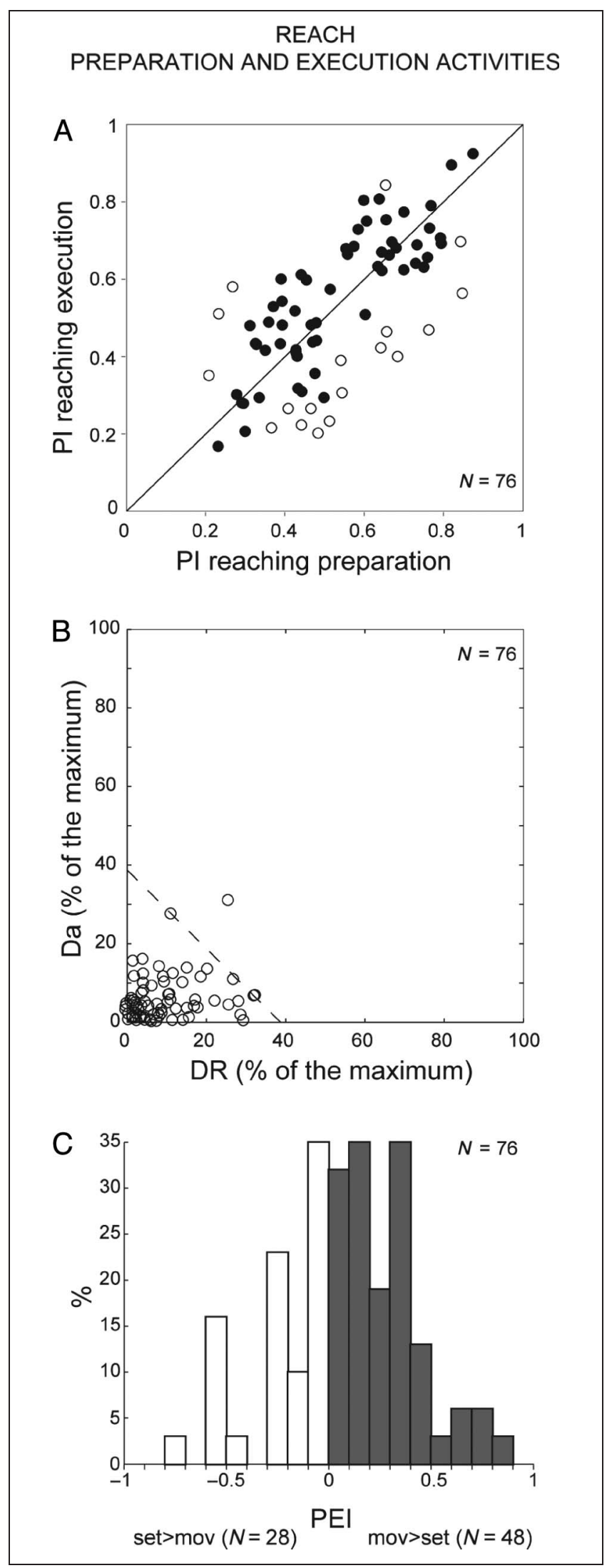

Table 2. Neurons ( $N$ and \%) Modulated by Vergence and/or Version during rSET

\begin{tabular}{lccccc}
\hline Type & Version & Vergence & Both & None & Total \\
\hline Gaze & $6(15 \%)$ & $14(34 \%)$ & $21(51 \%)$ & $0(0 \%)$ & $41(100 \%)$ \\
Set & $3(11 \%)$ & $10(37 \%)$ & $13(48 \%)$ & $1(4 \%)$ & $27(100 \%)$ \\
Gaze/Set & $11(15 \%)$ & $11(15 \%)$ & $31(45 \%)$ & $18(25 \%)$ & $71(100 \%)$ \\
Total & $20(14 \%)$ & $35(25 \%)$ & $65(47 \%)$ & $19(14 \%)$ & 139 \\
\hline
\end{tabular}

We then looked at the congruency of spatial preference between reach preparation and execution. Figure 8B shows that we found a similar spatial trend: The shift between preferred positions during reaching preparation and execution was not statistically different from zero for all the cells but one. In other words, the estimated preferred position of cells (vector endpoint; see Methods) did not change significantly across the two epochs. We quantitatively evaluated the congruence of tuning between movement preparation and execution by comparing the sign of the significant linear regression coefficients of epochs rSET and MOV. This comparison was possible only for cells whose activity fitted significantly the linear regression model $(N=36)$. We found that in the overwhelming majority of these cells $(34 / 36,94 \%)$ the spatial tunings were congruent. These results strongly support the view that the activity of these cells during reaching preparation actually encodes the movement plan and predicts the cell's behavior observed during movement execution.

As a further aspect of the comparison between reaching preparation and execution, we checked whether cells tuned in both epochs were more involved in the preparation or in the execution of arm movements. With this in mind, we calculated a PEI that assumed values equal to zero when the cell was equally involved in the two processes, positive values when the cell was more involved

Figure 8. Characterization of cells modulated by planning and execution of reaching: spatial selectivity, preferred positions, and modulation index. (A) Scatterplot of PIs of reach preparation (rSET, $x$ axis) and execution (MOV, $y$ axis). Each dot represents one neuron $(N=76)$. The black dots indicate neurons whose bootstrap-estimated confidence intervals cross the diagonal, the white dots indicate those whose bootstrap-estimated confidence intervals do not cross the diagonal. The majority of V6A cells are equally strongly tuned in reach preparation and execution. (B) Distribution of the shifts of the preferred position between reaching preparation and execution; $x$ axis, radial shift (difference between the radial coordinates of the preferred positions during reaching preparation and execution) expressed as percentage of the maximum shift; $y$ axis, angular shift (difference between the angular coordinates of the preferred positions during reaching preparation and execution) expressed as percentage of the maximum shift. Equation of dashed line is $R+\alpha=0.39$. Note that the spatial tunings during reaching preparation and execution are congruent for almost all cells. (C) Distribution of PEI (see Methods). Columns depicted in gray represent the frequency of cells with positive PEI, and in white, negative PEI. The majority of cells showed positive PEI, thus a prevalent execution-related discharge. 
in execution, and negative values when it was more involved in movement preparation (see Methods). Although in many neurons the PEI assumed values within \pm 0.5 (Figure 8C), confirming the convergence of preparation and execution signals upon single V6A cells, the majority (48/76, 63\%) of cells modulated during movement preparation and execution had positive PEI, that is, they showed predominantly execution-related activity.

\section{Timing of Modulation of Reaching Preparation Activity}

If the activity during reaching preparation was fully related to the movement plan, its onset should be strictly tied to the onset of movement. With this in mind, we checked the onset time of activity modulation during reaching preparation by comparing the activities during delay before button release in Reaching and Detection tasks, respectively. The comparison was carried out by performing a sliding window $t$ test that started from the go signal and went backwards (see Methods and Figure 9A). In the cells that showed a significant difference in activity at the go signal, we considered the onset of activity modulation as the time of the first bin where the $t$ test of comparison was no more significant. The analysis was applied to both Set and Gaze/Set cells.

In many cases, the discharge rate of cells showed high variability, and during reaching preparation, it continuously
Figure 9. Timing of modulation of reaching preparation activity. (A-C) Sliding window firing rate analysis. (A) Scheme of the method used to calculate the onset of the difference between the delay activity of Reaching and Detection tasks. To this purposes, some exemplifying, representative trials of the two tasks (Reaching trials, shown inside the top box, and Detection trials, shown inside the bottom box) are reported. The black square indicates the sliding window of $100 \mathrm{msec}$ where the algorithm counted the spikes and performed the Student's $t$ test. This window was shifted by 10 msec in the $1700 \mathrm{msec}$ before the go signal (zero). The arrowhead on top indicates the Onset that the algorithm found in case of these representative trials. (B, C) Distribution histograms (bin size $=160 \mathrm{msec}$ ) of the onsets of Set cells (B) and Gaze/Set cells (C). Horizontal axis: time with respect to the go signal; vertical axis: percentage of cells (with respect to the total population of Set + Gaze/ Set cells). Note that Set cells are always excited and time-locked to the go signal, whereas Gaze/ Set cells can be excited (and time-locked to the go signal) or inhibited (and temporally disengaged from the motor act). (D, E) Averaged SDFs analysis. Percentages of Set (D), Gaze/Set (E) cells with an early (white), intermediate (gray), or late (black) onset of the difference between Reaching and Detection activity. Gaze/Set cells were divided in excited (right) and inhibited (left). This analysis confirms in a higher number of cells the trend observed for data shown in A-C: Excited cells showed more late onsets, close to the go signal, whereas the inhibited cells showed early onsets more disengaged from the motor act.

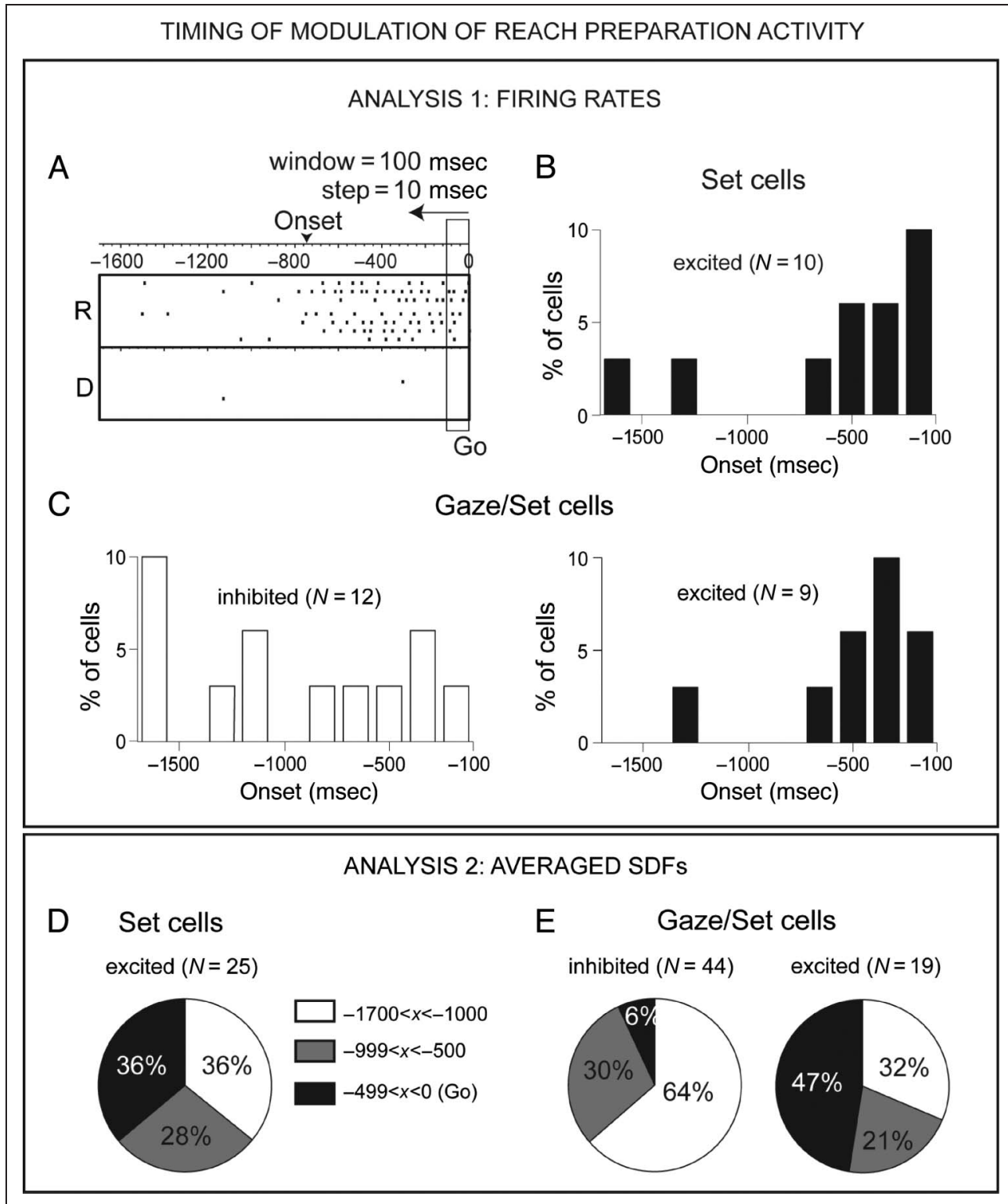

A

C
E Gaze/Set cells 
fluctuated above and below the level of activity observed in the same cell in Detection task. This behavior indicates a lack of clear influence of the impending movement on cell activity. In other cells, instead, during reaching preparation the discharge rate became suddenly and consistently higher (excitation) or lower (inhibition) than in Detection task. Figure 9A shows an example of this behavior in representative reaching (R) and detection (D) trials of a V6A cell. The cell was almost silent during fixation in Detection trials whereas suddenly started to discharge at a certain point during arm movement preparation. The activity was significantly different $(p<.05)$ in the first 10 windows near the go signal (black rectangle in Figure 9A represents the first window) as well as in several other windows backward from the go signal. The first time window where the $t$ test was not significant $(p>.05)$ was at $750 \mathrm{msec}$ before the go signal (arrowhead in Figure 9A). This was considered as the onset of activity change between Reaching and Detection trials. Figure 9 (B, C) shows the distributions of these onsets of activity modulation (excitations or inhibitions) in 31 cells. By analyzing separately the activity of Set cells and that of Gaze/Set cells, we realized that the former (Figure 9B) were all excited by the arm movement preparation whereas the latter (Figure 9C) could be inhibited or excited. The excitation in Gaze/Set cells mainly started less than 500 msec before the go signal, as in the Set cells, whereas inhibition showed a quite uniform distribution of time onsets. In other words, the Set cells and the excited Gaze/Set cells were time-locked to the upcoming arm movement, whereas the inhibited Gaze/Set cells were disengaged from the impending motor act.

This type of analysis produced results with a high temporal resolution but was quite sensitive to the noise, and only a minority of cells fulfilled the criteria to obtain reliable results. Thus, to check the onset of activity change between Reaching and Detection trials in a higher number of cells, we used the sliding window approach on SDFs at a single cell level. This approach has a lower temporal resolution than the first type of analysis but could be applied on the vast majority of recorded cells (88/98, 90\%). The distribution of the onsets for Set, excited Gaze/Set, and inhibited Gaze/Set cells is shown in Figure 9D and E. As evident, Set (Figure 9D) and excited Gaze/Set cells (Figure 9E, right) had similar distributions of onsets, most of them starting close to the go signal. In contrast, inhibited Gaze/Set cells (Figure 9E, left) changed their activity mostly during the early phases of fixation, with only a few cells changing activity close to the go signal. So, this second approach confirms in a larger group of cells the trend observed in the small sample of cells analyzed with the first, more selective method (Figure 9A-C).

\section{Activity Modulations for Execution of Arm Movements}

After steady fixation of the target, at the go signal, the monkeys executed arm reaching movements toward the

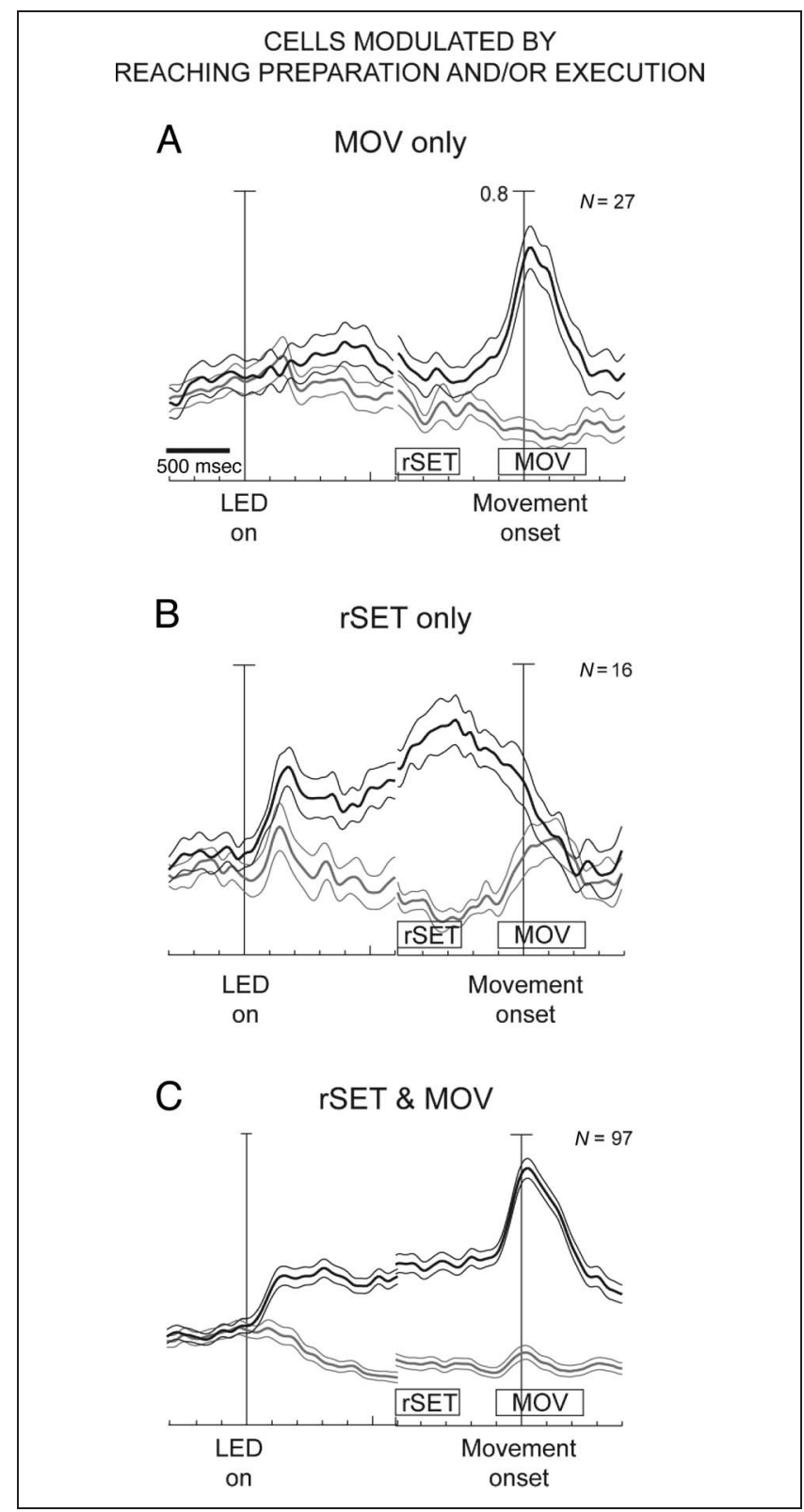

Figure 10. Population activity of cells modulated by movement execution and/or preparation. (A-C) Average normalized SDFs of cells modulated only by movement execution (A), only by movement preparation (B), and by both (C). For each cell category, the average SDFs for conditions with maximal (black) and minimal (gray) discharge are plotted. Conditions were sorted by activity in MOV (A and C, right) and during rSET (B and C, left). Scale bar in all SDF plots: $80 \%$ of normalized activity. Alignment on LED and Movement onsets. Other conventions as in Figure 6.

target. Both animals showed higher arm movement times when targets were located contralaterally and in the far space [mean movement time for ipsilateral space: $383.6 \pm$ $57.6 \mathrm{msec}$, for contralateral space: $459.5 \pm 108.8 \mathrm{msec}$ (ANOVA, $p<.05$ ); mean movement time for near space: $378.6 \pm 54.3 \mathrm{msec}$; for far space: $513.5 \pm 100.2 \mathrm{msec}$ (ANOVA, $p<.05$ )].

To have a quantitative estimate of whether V6A is more involved in execution or in preparation of arm move- 
ments, we compared the incidence of tuning during epochs MOV and rSET in the whole population of recorded neurons. Of 162 cells, 97 (60\%) were spatially tuned (one-way ANOVA, $p<.05$ ) in both MOV and rSET, 27 (17\%) were spatially tuned only in MOV ("MOV only" cells), 16 (10\%) only during rSET ("rSET only" cells), and the remaining 22 (13\%) in none of the above. Figure 10 shows the averaged population activities (SDF) of these cells. Figure 10A shows the behavior of "MOV only" cells. The two curves relative to preferred and nonpreferred target positions were superimposed during the delay period. They started to diverge at the beginning of MOV epoch, 200 msec before the onset of reaching movement, and continued to be well apart till the end of the movement. The cells with spatial tuning during only rSET and during both rSET and MOV (Figure 10B and C) - as expected-had a different time course of modulation. The SDF curves representing the positions that evoked the highest and lowest activities became well separated at the time of the saccade that brought the future reaching target on the fovea and during rSET the largest difference between the two curves was observed in the "SET only" cells (Figure 10B). In "rSET and MOV" cells, the difference between best and worst discharges was higher in MOV than in rSET.

\section{DISCUSSION}

To investigate the neural correlates of reach preparation in V6A, we compared the activity of single V6A neurons in two conditions: while the monkey was fixating a target waiting for the go signal to reach the target and while it was fixating a target without being allowed to perform any reach toward it. Because in the first task, but not in the second, the animal was preparing an arm reaching movement, the difference in activity between the two tasks should be attributed to the preparation of impending arm movement. The different activity in the two tasks cannot be ascribed to the visual stimulation evoked by target appearance because it was identical in both detection and reaching trials. Notice, also, that delay activity in our task was not affected by working memory processing because the fixated LED that specified direction and distance of target to be reached out was always turned on.

We found that about $44 \%$ of cells were influenced by both target location and reach preparation (Gaze/Set cells) and that $25 \%$ of cells encoded target location regardless of whether the target would be actually reached out or not (Gaze cells). As relatively few V6A cells (17\%) encoded reach preparation solely (Set cells), we suggest that V6A is more involved in encoding the gaze/spatial signals concerning visual targets than the planning of reaching actions. This finding supports the general view that processing in V6A is pivotal for encoding target location (Breveglieri, Hadjidimitrakis, Bosco, et al., 2012; Davare, Zénon, Pourtois, Desmurget, \& Olivier, 2012; Vesia \&
Crawford, 2012; Crawford, Henriques, \& Medendorp, 2011; Galletti \& Fattori, 2002; Galletti, Battaglini, \& Fattori, 1993).

We are aware that the task configuration we used has some limitations in studying the neural correlates of planning arm reaching movement (see Introduction). All task designs, including ours, have an artificial structure aimed to standardize the studied process, in which virtually only one variable at a time is changed. This is what we tried to do here. In the following sections, we will provide some interpretations of current results.

\section{Excitation and Inhibition of Neural Activity during Reaching Preparation}

During preparation of reaching movement, cell activity could increase or decrease with respect to the activity during fixation in Detection task. In the first case (excited cells), the increase of discharge was time-locked to the onset of impending arm movement (Figure 9B, C right plot, D, E right plot). This suggests that, during delay before arm movement, the activity was related to the planning of reaching, a view strongly supported by the direct input that V6A receives from PMd (Gamberini et al., 2009; Shipp, Blanton, \& Zeki, 1998). The congruency of spatial tuning of the discharge before and during movement execution further supports this view. V6A appears to be able to code preparation (and subsequent execution) of arm movements similarly to many other PPC areas (e.g., Bhattacharyya, Musallam, \& Andersen, 2009; BattagliaMayer et al., 2001; Snyder et al., 2000; Barash et al., 1991; Crammond \& Kalaska, 1989).

In the cells inhibited during reaching preparation, the onset of activity modulation was highly variable and not time-locked to the onset of movement (Figure 9C left plot, E left plot). We suggest that in these cells the tuning of activity during reach preparation did not reflect movement planning per se, but it was likely related to other processes. One of these could be an increase of alertness level evoked by the presence of reaching target. An attention/alertness effect is expected also to be present when the fixated target is not going to be reached, but it is plausible that the Reaching task requires higher attention/alertness than the Detection task, because the target becomes the focus of a spatially targeted movement. In line with this hypothesis, it was reported that V6A receives directly from area 46 (Gamberini et al., 2009), which is known to be involved in attentional control (IchiharaTakeda \& Funahashi, 2007; Watanabe \& Funahashi, 2007) and that V6A cells are often inhibited when the animal increased its alertness (see Figures 16 and 17 of Galletti et al., 1996). Alternatively, the higher discharge rates in Detection trials may be related to a gaze position signal suppressed during arm movement planning. Or, it may be because of the fact that in detection trials, but not in reaching trials, the monkeys prepared a break of fixation when the go signal appears. This might explain 
the discharges of cells spatially tuned in fSET but not in rSET. This phenomenon is similar to that observed in area LIP, where some neurons showed a buildup of activity before breaks of fixation (Ben Hamed \& Duhamel, 2002). As attention and eye movements are tightly associated, the upcoming eye movement would inevitably cause a shift of attention. Therefore, the higher firing in the detection trials could be related to attentional reorienting. This hypothesis is supported by a recent study where the human homolog of area V6A has been demonstrated to be involved in encoding reorienting of attention (Ciavarro et al., 2013).

The presence of neurons inhibited and other excited by reach preparation represent a reciprocal pattern similar to that reported in the pre-SMA and in the rostral ACC in humans (called "increasing" and "decreasing" neurons; Fried, Mukamel, \& Kreiman, 2011). It has been proposed that neurons inhibited during reach preparation might withhold actions until they become appropriate. It could be the same for V6A cells, because they are inhibited during fixation before reaching, when the movement is withheld until the go signal occurs. Competitive interaction between excited and inhibited neurons could provide a circuit for resolving whether to act or withhold action, as proposed in lateral premotor cortex for decisions between alternative stimulus-driven actions (Cisek, 2007).

As mentioned in the Introduction, the preparatory activity during reaches has been studied in area PE and in PMd with task configurations similar to ours (Kalaska \& Crammond, 1995; Boussaoud \& Wise, 1993). In the Kalaska's study, the monkeys were instructed to reach targets in one task condition (go trials), whereas in the other (no-go trials) a different illumination of the target instructed them to withhold their movement (Kalaska \& Crammond, 1995). It was observed that during the delay period the activity of PE neurons showed a similar directional selectivity in both types of trials, whereas PMd neurons showed different responses that were more tightly associated with reaching preparation. The modulations observed in V6A in this study are similar to those found in PMd, consistent with the strong connections between the two areas (Gamberini et al., 2009). PMd neurons also show another functional property that could explain the firing pattern of V6A neurons in this study. In PMd cortex it was observed that, when several targets are used to cue the same movement, a condition very similar to our Detection task, the activity of neurons is modulated by the spatial location of target (Boussaoud \& Wise, 1993; di Pellegrino \& Wise, 1993). So, visuospatial processing could have a strong effect on both PMd and V6A activity.

\section{The Functional Role of Area V6A: Comparison with Area PRR and with Human Studies}

Many studies in the last 15 years have demonstrated that area PRR, located nearby V6A (see Figure 1), is involved in planning reaching movements. In their seminal work, Andersen and coworkers (Snyder et al., 1997) revealed for the first time the neural signature of a motor plan in PPC by showing that the majority of PRR neurons had sustained elevated activity during arm movement preparation. Similarly, we found V6A neurons showing a sustained activity during reaching preparation that correlates with the discharge during reaching execution. However, the cells excited by reach plan in V6A (about 30\% in this study, considering the excited Set and Gaze/Set cells) were fewer than those reported in PRR (from 73\%, Chang, Dickinson, \& Snyder, 2008, to 86\%, Hwang \& Andersen, 2012). It should be noted that experimental conditions were slightly different in the studies of the two PPC areas. In PRR studies, gaze location was dissociated from reaching target location, that is, the target was typically located in a peripheral retinal position, whereas in this study the two positions overlapped, because the animal only performed foveal reaching. As the relative position of gaze and reaching target has been shown to be an important factor influencing PPC cells (Mullette-Gillman, Cohen, \& Groh, 2005, 2009; Marzocchi et al., 2008; Buneo, Jarvis, Batista, \& Andersen, 2002; Batista, Buneo, Snyder, \& Andersen, 1999), with V6A cells strongly activated for peripheral reaching that did not respond when reaches were performed toward a foveated target (Marzocchi et al., 2008), this difference in task design could at least in part explain the difference in percentage of cells modulated by reach planning in the two areas. Another crucial difference is that in the Andersen studies, the visual signal provided instructions concerning which type of movement should be planned (arm and/or eye), whereas in the current study the instruction tells the animal whether it needs to plan a reach or to release a key. Apart from all these differences, overall the current results show that V6A is less involved in reaching preparation than PRR. We argue that V6A is likely to be more involved in the coding of reaching movement execution than in planning, a hypothesis supported by many of the current data: (i) V6A contains more cells modulated during movement execution than during movement planning; (ii) the majority of cells tuned for both reach planning and execution showed a stronger modulation for execution (Figure 8C); (iii) the activity modulation of cells tuned during both movement preparation and execution was higher during the execution of movement (Figure 10C).

We argue that V6A encodes mainly the spatial signals to localize targets in 3-D space whereas PRR encodes mainly the intention to reach. This view is in agreement with what was found in human PPC, where more posterior regions (including the putative homologue of area V6A; see Pitzalis et al., 2013) were less activated during reaching preparation than more anterior areas (including the putative homologue of PRR; see Galati et al., 2011). A similar gradient of selectivity seems to exist in monkey 
PPC, with the more anterior area PRR being more actionspecific than area V6A.

Several human fMRI studies have found that reaching preparation and/or execution activate a region putatively homologue to monkey area V6A (Galati et al., 2011; Gallivan, McLean, Valyear, Pettypiece, \& Culham, 2011; Cavina-Pratesi et al., 2010; Filimon, Nelson, Hagler, \& Sereno, 2007). A huge amount of fMRI studies have also reported activity in the medial PPC (likely including the homolog of monkey area V6A) during the preparation and execution of simple finger-pointing movements (Bernier, Cieslak, \& Grafton, 2012; Beurze, Toni, Pisella, \& Medendorp, 2010; Beurze, de Lange, Toni, \& Medendorp, 2007, 2009; Medendorp, Goltz, Crawford, \& Vilis, 2005; Prado et al., 2005; Simon et al., 2004; Medendorp, Goltz, Vilis, \& Crawford, 2003; Connolly, Goodale, DeSouza, Menon, \& Vilis, 2000; DeSouza et al., 2000; Kertzman, Schwarz, Zeffiro, \& Hallett, 1997). Our results are consistent with the above studies, supporting the view of the existence of common neural mechanisms for reach planning and execution control in human and nonhuman primate medial PPC areas.

Present and previous results can help understanding the functional role played by area V6A. Besides a third of neurons able to encode the reach plan, many V6A neurons are able to encode the spatial location of foveated reaching targets because of the modulating effect of gaze signal (Gaze cells and Gaze/Set cells), and in the large majority of V6A neurons the strongest modulations do occur during movement execution. Previous data showed that V6A neurons were also able to encode nonfoveated visual targets because of the gaze modulation of visual responses (Galletti et al., 1995) and, in some cases, to the existence of cells directly encoding spatial locations in headcentered frame of reference (Galletti et al., 1993; see, for a review, Galletti \& Fattori, 2002). The previous studies also showed that many V6A neurons are able to encode the direction of arm reaching movements (e.g., Fattori et al., 2005) and that some of them are modulated by covert spatial attention (Galletti et al., 2010). All together these data suggest that V6A is involved in all the processes needed to control an arm reaching movement, that is, target selection and location in space, planning of arm movement, and movement execution. If we add the fact that several V6A neurons are able to encode wrist orientation and grip formation (Fattori et al., 2009, 2010), the view that the medial posterior parietal area V6A is involved in the control of prehension as a whole turns out further supported.

\section{Acknowledgments}

This work was supported by EU FP7-IST-217077-EYESHOTS, Ministero dell'Università e della Ricerca (Italy), and Fondazione del Monte di Bologna e Ravenna (Italy). We thank N. Marzocchi and G. Placenti for setting up the experimental apparatus, F. Bertozzi and A. Bosco for helping in recordings, and M. Gamberini and $\mathrm{L}$. Passarelli for the anatomical reconstructions.
Reprint requests should be sent to Prof. Patrizia Fattori, Department of Pharmacy and Biotechnology, University of Bologna, Piazza di Porta S. Donato, 2, 40126 Bologna, Italy, or via e-mail: patrizia.fattori@unibo.it.

\section{REFERENCES}

Andersen, R. A., \& Buneo, C. A. (2002). Intentional maps in posterior parietal cortex. Annual Review of Neuroscience, 25, 189-220.

Andersen, R. A., \& Cui, H. (2009). Intention, action planning, and decision making in parietal-frontal circuits. Neuron, 63, 568-583.

Baldauf, D., \& Deubel, H. (2008). Visual attention during the preparation of bimanual movements. Vision Research, 48, 549-563.

Baldauf, D., Wolf, M., \& Deubel, H. (2006). Deployment of visual attention before sequences of goal-directed hand movements. Vision Research, 46, 4355-4374.

Barash, S., Bracewell, R. M., Fogassi, L., Gnadt, J. W., \& Andersen, R. A. (1991). Saccade-related activity in the lateral intraparietal area. II. Spatial properties. Journal of Neurophysiology, 66, 1109-1124.

Batista, A. P., Buneo, C. A., Snyder, L. H., \& Andersen, R. A. (1999). Reach plans in eye-centered coordinates. Science, 285, 257-260.

Batista, A. P., Santhanam, G., Yu, B. M., Ryu, S. I., Afshar, A., \& Shenoy, K. V. (2007). Reference frames for reach planning in macaque dorsal premotor cortex. Journal of Neurophysiology, 98, 966-983.

Battaglia-Mayer, A., Ferraina, S., Genovesio, A., Marconi, B., Squatrito, S., Molinari, M., et al. (2001). Eye-hand coordination during reaching. II. An analysis of the relationships between visuomanual signals in parietal cortex and parieto-frontal association projections. Cerebral Cortex, 11, 528-544.

Battaglia-Mayer, A., Mascaro, M., \& Caminiti, R. (2007). Temporal evolution and strength of neural activity in parietal cortex during eye and hand movements. Cerebral Cortex, 17, 1350-1363.

Ben Hamed, S., \& Duhamel, J. R. (2002). Ocular fixation and visual activity in the monkey lateral intraparietal area. Experimental Brain Research, 142, 512-528.

Bernier, P. M., Cieslak, M., \& Grafton, S. T. (2012). Effector selection precedes reach planning in the dorsal parietofrontal cortex. Journal of Neurophysiology, 108, 57-68.

Beurze, S. M., de Lange, F. P., Toni, I., \& Medendorp, W. P. (2007). Integration of target and effector information in the human brain during reach planning. Journal of Neurophysiology, 97, 188-199.

Beurze, S. M., de Lange, F. P., Toni, I., \& Medendorp, W. P. (2009). Spatial and effector processing in the human parietofrontal network for reaches and saccades. Journal of Neurophysiology, 101, 3053-3062.

Beurze, S. M., Toni, I., Pisella, L., \& Medendorp, W. P. (2010). Reference frames for reach planning in human parietofrontal cortex. Journal of Neurophysiology, 104, 1736-1745.

Bhattacharyya, R., Musallam, S., \& Andersen, R. A. (2009). Parietal reach region encodes reach depth using retinal disparity and vergence angle signals. Journal of Neurophysiology, 102, 805-816.

Bosco, A., Breveglieri, R., Chinellato, E., Galletti, C., \& Fattori, P. (2010). Reaching activity in the medial posterior parietal cortex of monkeys is modulated by visual feedback. Journal of Neuroscience, 30, 14773-14785.

Boussaoud, D., \& Wise, S. P. (1993). Primate frontal cortex: Effects of stimulus and movement. Experimental Brain Research, 95, 28-40. 
Breveglieri, R., Hadjidimitrakis, K., Bosco, A., Sabatini, S. P., Galletti, C., \& Fattori, P. (2012). Eye position encoding in three-dimensional space: Integration of version and vergence signals in the medial posterior parietal cortex. Journal of Neuroscience, 32, 159-169.

Breveglieri, R., Hadjidimitrakis, K., Dal Bò, G., Bertozzi, F., Bosco, A., Galletti, C., et al. (2012). Reach plan or gaze modulation? Multiple aspects of delay activity in area V6A of the monkey medial posterior parietal cortex (Program No. 829.04. 2012 Neuroscience Meeting Planner, Online). New Orleans, LA: Society for Neuroscience.

Buneo, C. A., \& Andersen, R. A. (2006). The posterior parietal cortex: Sensorimotor interface for the planning and online control of visually guided movements. Neuropsychologia, 44, 2594-2606.

Buneo, C. A., Jarvis, M. R., Batista, A. P., \& Andersen, R. A. (2002). Direct visuomotor transformations for reaching. Nature, 416, 632-636.

Cavina-Pratesi, C., Monaco, S., Fattori, P., Galletti, C., McAdam, T. D., Quinlan, D. J., et al. (2010). Functional magnetic resonance imaging reveals the neural substrates of arm transport and grip formation in reach-to-grasp actions in humans. Journal of Neuroscience, 30, 10306-10323.

Chang, S. W., Dickinson, A. R., \& Snyder, L. H. (2008). Limb-specific representation for reaching in the posterior parietal cortex. Journal of Neuroscience, 28, 6128-6140.

Ciavarro, M., Ambrosini, E., Tosoni, A., Committeri, G., Fattori, P., \& Galletti, C. (2013). rTMS of medial parieto-occipital cortex interferes with attentional reorienting during attention and reaching tasks. Journal of Cognitive Neuroscience, 25, 1453-1462.

Cisek, P. (2007). Cortical mechanisms of action selection: The affordance competition hypothesis. Philosophical Transactions of the Royal Society of London, Series B, Biological Sciences, 362, 1585-1599.

Colby, C. L., \& Duhamel, J. R. (1991). Heterogeneity of extrastriate visual areas and multiple parietal areas in the macaque monkey. Neuropsychologia, 29, 517-537.

Confais, J., Kilavik, B. E., Ponce-Alvarez, A., \& Riehle, A. (2012). On the anticipatory precue activity in motor cortex. Journal of Neuroscience, 32, 15359-15368.

Connolly, J. D., Goodale, M. A., DeSouza, J. F., Menon, R. S., \& Vilis, T. (2000). A comparison of frontoparietal fMRI activation during anti-saccades and anti-pointing. Journal of Neurophysiology, 84, 1645-1655.

Crammond, D. J., \& Kalaska, J. F. (1989). Neuronal activity in primate parietal cortex area 5 varies with intended movement direction during an instructed-delay period. Experimental Brain Research, 76, 458-462.

Crawford, J. D., Henriques, D. Y., \& Medendorp, W. P. (2011). Three-dimensional transformations for goal-directed action Annual Review of Neuroscience, 34, 309-331.

Davare, M., Zénon, A., Pourtois, G., Desmurget, M., \& Olivier, E. (2012). Role of the medial part of the intraparietal sulcus in implementing movement direction. Cerebral Cortex, 22, 1382-1394.

DeSouza, J. F., Dukelow, S. P., Gati, J. S., Menon, R. S., Andersen, R. A., \& Vilis, T. (2000). Eye position signal modulates a human parietal pointing region during memory-guided movements. Journal of Neuroscience, 20, 5835-5840.

di Pellegrino, G., \& Wise, S. P. (1993). Visuospatial versus visuomotor activity in the premotor and prefrontal cortex of a primate. Journal of Neuroscience, 13, 1227-1243.

Eskandar, E. N., \& Assad, J. A. (1999). Dissociation of visual, motor and predictive signals in parietal cortex during visual guidance. Nature Neuroscience, 2, 88-93.
Fattori, P., Breveglieri, R., Marzocchi, N., Filippini, D., Bosco, A., \& Galletti, C. (2009). Hand orientation during reach-to-grasp movements modulates neuronal activity in the medial posterior parietal area V6A. Journal of Neuroscience, 29, 1928-1936.

Fattori, P., Gamberini, M., Kutz, D. F., \& Galletti, C. (2001) "Arm-reaching" neurons in the parietal area V6A of the macaque monkey. European Journal of Neuroscience, 13, 2309-2313.

Fattori, P., Kutz, D. F., Breveglieri, R., Marzocchi, N., \& Galletti, C. (2005). Spatial tuning of reaching activity in the medial parieto-occipital cortex (area V6A) of macaque monkey. European Journal of Neuroscience, 22, 956-972.

Fattori, P., Raos, V., Breveglieri, R., Bosco, A., Marzocchi, N., \& Galletti, C. (2010). The dorsomedial pathway is not just for reaching: Grasping neurons in the medial parieto-occipital cortex of the macaque monkey. Journal of Neuroscience, 30, 342-349.

Filimon, F., Nelson, J. D., Hagler, D. J., \& Sereno, M. I. (2007). Human cortical representations for reaching Mirror neurons for execution, observation, and imagery. Neuroimage, 37, 1315-1328.

Fried, I., Mukamel, R., \& Kreiman, G. (2011). Internally generated preactivation of single neurons in human medial frontal cortex predicts volition. Neuron, 69, $548-562$

Galati, G., Committeri, G., Pitzalis, S., Pelle, G., Patria, F., Fattori, P., et al. (2011). Intentional signals during saccadic and reaching delays in the human posterior parietal cortex. European Journal of Neuroscience, 34, 1871-1885.

Galletti, C., Battaglini, P. P., \& Fattori, P. (1993). Parietal neurons encoding spatial locations in craniotopic coordinates. Experimental Brain Research, 96, 221-229.

Galletti, C., Battaglini, P. P., \& Fattori, P. (1995). Eye position influence on the parieto-occipital area PO (V6) of the macaque monkey. European Journal of Neuroscience, 7, 2486-2501.

Galletti, C., Breveglieri, R., Lappe, M., Bosco, A., Ciavarro, M., \& Fattori, P. (2010). Covert shift of attention modulates the ongoing neural activity in a reaching area of the macaque dorsomedial visual stream. PLoS One, 5, e15078.

Galletti, C., \& Fattori, P. (2002). Posterior parietal networks encoding visual space. In H. O. Karnath, A. D. Milner, \& G. Vallar (Eds.), The cognitive and neural bases of spatial neglect (pp. 59-69). New York: Oxford University Press.

Galletti, C., Fattori, P., Battaglini, P. P., Shipp, S., \& Zeki, S. (1996). Functional demarcation of a border between areas $\mathrm{V} 6$ and $\mathrm{V} 6 \mathrm{~A}$ in the superior parietal gyrus of the macaque monkey. European Journal of Neuroscience, 8, 30-52.

Galletti, C., Fattori, P., Gamberini, M., \& Kutz, D. F. (1999). The cortical visual area V6: Brain location and visual topography. European Journal of Neuroscience, 11, 3922-3936.

Galletti, C., Fattori, P., Kutz, D. F., \& Gamberini, M. (1999). Brain location and visual topography of cortical area V6A in the macaque monkey. European Journal of Neuroscience, 11, 575-582.

Galletti, C., Kutz, D. F., Gamberini, M., Breveglieri, R., \& Fattori, P. (2003). Role of the medial parieto-occipital cortex in the control of reaching and grasping movements. Experimental Brain Research, 153, 158-170.

Gallivan, J. P., McLean, D. A., Valyear, K. F., Pettypiece, C. E., \& Culham, J. C. (2011). Decoding action intentions from preparatory brain activity in human parieto-frontal networks. Journal of Neuroscience, 31, 9599-9610.

Gamberini, M., Galletti, C., Bosco, A., Breveglieri, R., \& Fattori, P. (2011). Is the medial posterior parietal area V6A a single functional area? Journal of Neuroscience, 31, 5145-5157. 
Gamberini, M., Passarelli, L., Fattori, P., Zucchelli, M., Bakola, S., Luppino, G., et al. (2009). Cortical connections of the visuomotor parietooccipital area V6Ad of the macaque monkey. Journal of Comparative Neurology, 513, 622-642.

Gottlieb, J., \& Snyder, L. H. (2010). Spatial and non-spatial functions of the parietal cortex. Current Opinion in Neurobiology, 20, 731-740.

Hadjidimitrakis, K., Bertozzi, F., Breveglieri, R., Bosco, A., Galletti, C., \& Fattori, P. (2013). Common neural substrate for processing depth and direction signals for reaching in the monkey medial posterior parietal cortex. Cerebral Cortex. doi:10.1093/cercor/bht021.

Hadjidimitrakis, K., Breveglieri, R., Placenti, G., Bosco, A., Sabatini, S. P., \& Fattori, P. (2011). Fix your eyes in the space you could reach: Neurons in the macaque medial parietal cortex prefer gaze positions in peripersonal space. PLoS One, 6, e23335.

Hwang, E. J., \& Andersen, R. A. (2012). Spiking and LFP activity in PRR during symbolically instructed reaches. Journal of Neurophysiology, 107, 836-849.

Ichihara-Takeda, S., \& Funahashi, S. (2007). Activity of primate orbitofrontal and dorsolateral prefrontal neurons: Task-related activity during an oculomotor delayed-response task. Experimental Brain Research, 181, 409-425.

Kalaska, J. F., \& Crammond, D. J. (1995). Deciding not to go: Neuronal correlates of response selection in a go/nogo task in primate premotor and parietal cortex. Cerebral Cortex, 5, 410-428.

Kertzman, C., Schwarz, U., Zeffiro, T. A., \& Hallett, M. (1997). The role of posterior parietal cortex in visually guided reaching movements in humans. Experimental Brain Research, 114, 170-183.

Kutz, D. F., Fattori, P., Gamberini, M., Breveglieri, R., \& Galletti, C. (2003). Early- and late-responding cells to saccadic eye movements in the cortical area V6A of macaque monkey. Experimental Brain Research, 149, 83-95.

Kutz, D. F., Marzocchi, N., Fattori, P., Cavalcanti, S., \& Galletti, C. (2005). Real-time supervisor system based on trinary logic to control experiments with behaving animals and humans. Journal of Neurophysiology, 93, 3674-3686.

Luppino, G., Ben Hamed, S., Gamberini, M., Matelli, M., \& Galletti, C. (2005). Occipital (V6) and parietal (V6A) areas in the anterior wall of the parieto-occipital sulcus of the macaque: A cytoarchitectonic study. European Journal of Neuroscience, 21, 3056-3076.

Mardia, K. V. (1972). Statistics of directional data. London/ New York: Academic Press.

Marzocchi, N., Breveglieri, R., Galletti, C., \& Fattori, P. (2008). Reaching activity in parietal area V6A of macaque: Eye influence on arm activity or retinocentric coding of reaching movements? European Journal of Neuroscience, 27, 775-789.

Medendorp, W. P., Goltz, H. C., Crawford, J. D., \& Vilis, T. (2005). Integration of target and effector information in human posterior parietal cortex for the planning of action. Journal of Neurophysiology, 93, 954-962.
Medendorp, W. P., Goltz, H. C., Vilis, T., \& Crawford, J. D. (2003). Gaze-centered updating of visual space in human parietal cortex. Journal of Neuroscience, 23, 6209-6214.

Moody, S. L., \& Zipser, D. (1998). A model of reaching dynamics in primary motor cortex. Journal of Cognitive Neuroscience, 10, 35-45.

Mullette-Gillman, O. A., Cohen, Y. E., \& Groh, J. M. (2005). Eye-centered, head-centered, and complex coding of visual and auditory targets in the intraparietal sulcus. Journal of Neurophysiology, 94, 2331-2352.

Mullette-Gillman, O. A., Cohen, Y. E., \& Groh, J. M. (2009). Motor-related signals in the intraparietal cortex encode locations in a hybrid, rather than eye-centered reference frame. Cerebral Cortex, 19, 1761-1775.

Nakamura, K., Chung, H. H., Graziano, M. S., \& Gross, C. G. (1999). Dynamic representation of eye position in the parieto-occipital sulcus. Journal of Neurophysiology, 81, 2374-2385.

Pandya, D. N., \& Seltzer, B. (1982). Intrinsic connections and architectonics of posterior parietal cortex in the rhesus monkey. Journal of Comparative Neurology, 204, 196-210.

Pitzalis, S., Sereno, M. I., Committeri, G., Fattori, P., Galati, G., Tosoni, A., et al. (2013). The human homologue of macaque area V6A. Neuroimage, 82C, 517-530.

Prado, J., Clavagnier, S., Otzenberger, H., Scheiber, C., Kennedy, H., \& Perenin, M. T. (2005). Two cortical systems for reaching in central and peripheral vision. Neuron, 48, 849-858.

Rushworth, M. F., Nixon, P. D., \& Passingham, R. E. (1997). Parietal cortex and movement. I. Movement selection and reaching. Experimental Brain Research, 117, 292-310.

Shipp, S., Blanton, M., \& Zeki, S. (1998). A visuo-somatomotor pathway through superior parietal cortex in the macaque monkey: Cortical connections of areas V6 and V6A. European Journal of Neuroscience, 10, 3171-3193.

Simon, O., Kherif, F., Flandin, G., Poline, J. B., Rivière, D., Mangin, J. F., et al. (2004). Automatized clustering and functional geometry of human parietofrontal networks for language, space, and number. Neuroimage, 23, 1192-1202.

Snyder, L. H., Batista, A. P., \& Andersen, R. A. (1997). Coding of intention in the posterior parietal cortex. Nature, 386, 167-170.

Snyder, L. H., Batista, A. P., \& Andersen, R. A. (1998). Change in motor plan, without a change in the spatial locus of attention, modulates activity in posterior parietal cortex. Journal of Neurophysiology, 79, 2814-2819.

Snyder, L. H., Batista, A. P., \& Andersen, R. A. (2000). Intention-related activity in the posterior parietal cortex: A review. Vision Research, 40, 1433-1441.

Vesia, M., \& Crawford, J. D. (2012). Specialization of reach function in human posterior parietal cortex. Experimental Brain Research, 221, 1-18.

Watanabe, K., \& Funahashi, S. (2007). Prefrontal delay-period activity reflects the decision process of a saccade direction during a free-choice ODR task. Cerebral Cortex, 17(Suppl. 1), i88-i100. 